\title{
Polyharmonic Distortion Modelling Applied to Acoustic Characterisation of Peforates
}

\author{
Hans Bodén ${ }^{1}$ \\ Linné Flow Centre, MWL, Aeronautical and Vehicle Engineering, KTH, SE-10044 Stockholm, Sweden
}

\begin{abstract}
This paper discusses the possibility to apply polyharmonic distortion modelling, used for nonlinear characterisation of microwave systems, to acoustic characterisation of samples with non-linear properties such as perforates and other facing sheets used in aircraft engine liners and automotive mufflers. In some previous papers multi-port techniques using sinusoidal excitation for characterization of samples with non-linear properties were developed and experimentally tested. These techniques aimed at taking non-linear energy transfer between sound field harmonics into account. Essentially linear system identification theory was however used assuming that superposition applies and that the functions studied are analytical. Polyharmonic distortion modelling does not assume that the function relating waves incident and reflected or transmitted is analytic nor does it assume application of normal superposition. This technique is tested on experimental data obtained from measurements on a perforate mounted in a duct. The similarity to the previously developed nonlinear scattering matrix techniques is demonstrated. It is shown how the results obtained can be used to analyse nonlinear energy transfer to higher harmonics.
\end{abstract}

\section{Introduction}

$I$

$\mathrm{N}$ a number of papers experimental techniques for determining acoustic impedance and two-port data for perforates under non-linear conditions have been developed ${ }^{1-5}$. Experiments were made using both pure tone and random excitation and the relevant parameters controlling the non-linearity were discussed. In Ref. 1 a study of harmonic interaction effects using two-tone excitations was made and in Ref. 2 the study was extended to multi-tone excitation for different types of perforates. In Ref. 3 a study was made of using non-linear system identification techniques for this purpose. The effect of sample non-linearity when performing impedance tube measurements were studied in Ref. 4 along with an outline of multi-port techniques for characterization of samples with non-linear properties. These multi-port techniques were further developed and experimentally tested in the Ref.5. Many investigations of nonlinear effects occurring when high amplitude sound waves are incident on perforated plates or orifice plates have been published, see e.g., Ref. 6-14. It is generally agreed that the non-linear losses are associated with vortex shedding at the outlet side of the orifice or perforate openings ${ }^{11-14}$. The nonlinear multi-port techniques with sinusoidal excitation developed and tested in Ref. 4 and 5 aimed at taking non-linear energy transfer between sound field harmonics into account. Essentially linear system identification theory was however used assuming that superposition applies and that the functions studied are analytical. Polyharmonic distortion modelling ${ }^{15-20}$, used for studying the nonlinear properties of microwave systems, does not assume that the function relating waves incident and reflected or transmitted is analytic nor does it assume application of normal superposition. For many years socalled Virtual Network Analysers have been used to characterise linear properties of microwave systems. The resulting parameters, called S-parameters, are essentially equivalent to the scattering matrix parameters used to characterise in-duct acoustic two-ports ${ }^{21}$. The last twenty years there has been an effort to include nonlinear properties leading to the development of so-called Large Signal Network Analyser technology. The polyharmonic distortion (PHD) model used will be described in more detail in section III. The resulting matrix relation between incident and reflected and transmitted waves is sometimes called X-parameters. The description of the polyharmonic distortion modeling in this paper is largely based on the papers by Verspect et al ${ }^{17-19}$ and the article by $\mathrm{Vye}^{20}$.

\footnotetext{
${ }^{1}$ Associate Professor, MWL, AVE, KTH, SE-10044 Stockholm Sweden, AIAA Member. 1

American Institute of Aeronautics and Astronautics
} 


\section{Problem formulation}

The problem of interest is outlined in Fig. 1 where the sample under test is mounted in a duct with at least two microphones on each side. A loudspeaker mounted in a side branch provides acoustic excitation on side A where the acoustic loading can also be changed by varying the length of the termination pipe. The termination on side B can also be varied during the test. Only plane wave propagation is considered. If the incident sound wave to a perforate sample with non-linear characteristics is single frequency there will be reflected and transmitted waves at that frequency but also at other frequencies. The strongest harmonic interaction should be a transfer of signal energy to frequencies three times higher than the excitation frequency ${ }^{1-5}$. There will also be higher order odd terms, that is 5 , 7, 9 times higher than the excitation frequency. For the perforate samples studied here we will therefore consider only the odd harmonics of the excitation frequency.

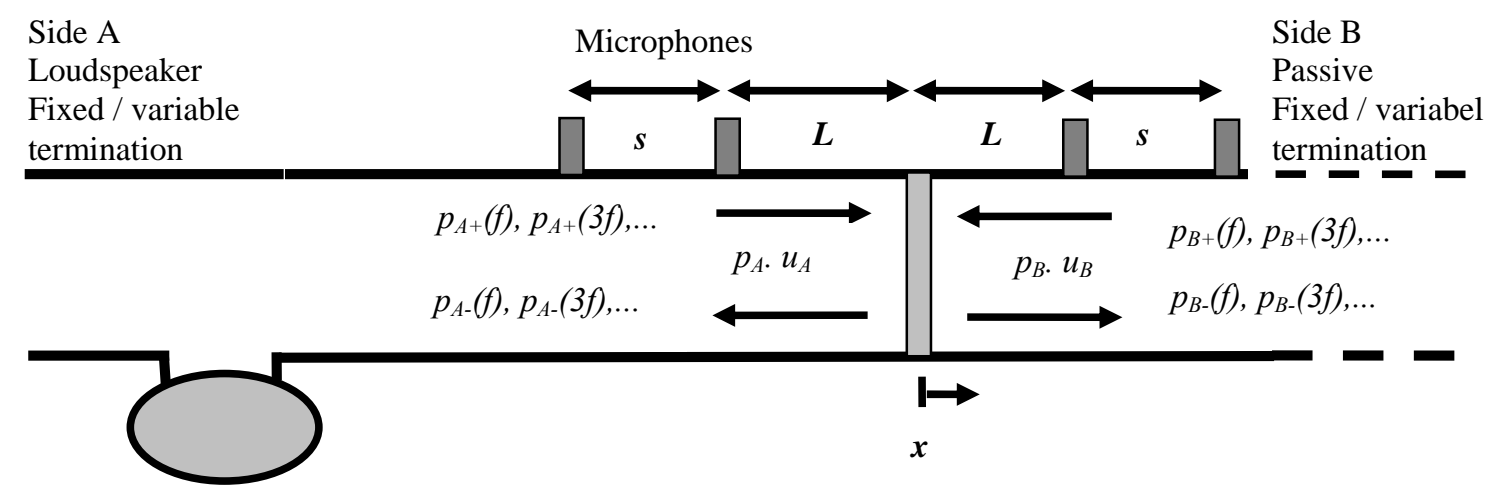

Figure 1. Two-port test rig.

It is assumed that although there is a sample with non-linear properties at $\mathrm{x}=0$ in the duct the non-linearity is only local and linear theory applies for the sound propagation on side A and B of the sample. The plane wave sound field on side A can then, for a frequency $n f$ where $n=1,3,5, .$. , be expressed as

$$
\begin{gathered}
p_{A}(x, n f)=p_{A+}(n f) \cdot e^{-j k_{n} x}+p_{A-}(n f) \cdot e^{j k_{n} x}=p_{A+}(n f) \cdot\left(e^{-j k_{n} x}+R_{A}(n f) \cdot e^{j k_{n} x}\right), \\
\rho c u_{A}(x, n f)=p_{A+}(n f) \cdot e^{-j k_{n} x}-p_{A-}(n f) \cdot e^{j k x}=p_{A+}(n f) \cdot\left(e^{-j k_{n} x}-R_{A}(n f) \cdot e^{j k_{n} x}\right),
\end{gathered}
$$

where $\rho$ is the density of air, c is the speed of sound, $p_{A}(x, n f)$ is the sound pressure at $x, u_{A}(x, n f)$ is the particle velocity at $x, p_{A^{+}}(n f)$ is the amplitude of the pressure wave in the positive $x$ direction, $p_{A-}(n f)$ is the amplitude of the pressure wave in the negative $x$ direction, $R_{A}(n f)$ is the reflection coefficient at $x=0$ and $k$ is the wave number. Similarly on the B side we have

$$
\begin{gathered}
p_{B}(x, n f)=p_{B-}(n f) \cdot e^{-j k_{n} x}+p_{B+}(n f) \cdot e^{j k_{n} x}=p_{B-}(n f) \cdot\left(e^{-j k_{n} x}+R_{B}(n f) \cdot e^{j k_{n} x}\right), \\
\rho c u_{B}(x, n f)=p_{B-}(n f) \cdot e^{-j k_{n} x}-p_{B+}(n f) \cdot e^{j k_{n} x}=p_{B-}(n f) \cdot\left(e^{-j k_{n} x}-R_{B}(n f) \cdot e^{j k_{n} x}\right),
\end{gathered}
$$

$p_{B-}(n f)$ is now the amplitude of the pressure wave in the positive $x$ direction but away from the sample, $p_{B+}(n f)$ is the amplitude of the pressure wave in the negative $x$ direction (towards the sample) and $R_{B}(n f)$ is the reflection coefficient at $x=0$ towards the passive termination. By measuring the pressure at two positions in the duct we can decompose the sound field into the waves going in positive and negative $\mathrm{x}$-direction and determine the reflection coefficients $^{22-23}$. 


$$
\begin{aligned}
p_{A+}(n f) & =e^{-j k_{n} L} \frac{p_{A}(-L, n f)+p_{A}(-(L+s), n f) e^{j k_{n} s}}{1-e^{j 2 k_{n} s},} \\
p_{A-}(n f) & =e^{j k_{n} L} \frac{p_{A}(-L, n f)+p_{A}(-(L+s), n f) e^{-j k_{n} s}}{1-e^{-j 2 k_{n} s}}, \\
p_{B+}(n f) & =e^{-j k_{n} L} \frac{p_{B}(L, n f)+p_{B}((L+s), n f) e^{j k_{n} s}}{1-e^{j 2 k_{n} s}}, \\
p_{B-}(n f) & =e^{j k_{n} L} \frac{p_{B}(L, n f)+p_{B}((L+s), n f) e^{-j k_{n} s}}{1-e^{-j 2 k_{n} s}}, \\
R_{A}(n f) & =\frac{p_{A-}(n f)}{p_{A+}(n f)}, \quad \text { (9) } \quad R_{B}(n f)=\frac{p_{B+}(n f)}{p_{B-}(n f)},
\end{aligned}
$$

The sound power towards or away from the sample on sides A and B can now also be expressed as

$$
\begin{gathered}
W_{A+}(n f)=S \frac{\left|p_{A+}(n f)\right|^{2}}{\rho c}, \\
W_{B+}(n f)=S \frac{\left|p_{B+}(n f)\right|^{2}}{\rho c},
\end{gathered}
$$

$$
\begin{gathered}
W_{A-}(n f)=S \frac{\left|p_{A-}(n f)\right|^{2}}{\rho c}, \\
W_{B-}(n f)=S \frac{\left|p_{B-}(n f)\right|^{2}}{\rho c},
\end{gathered}
$$

where $\mathrm{S}$ is the duct cross section area.

The idea is now to use system identification techniques to find relations between the travelling wave components ar different frequencies on both sides of the sample. If this is successful we can for instance based on the expressions given above calculate the power generated, by nonlinear energy transfer, at each harmonic, not only for the terminations used for the measurements but for any termination on side A and B.

\section{Multi-port Models for Non-linear Samples}

The approach is to analyze the problem described in section II using system identification and multi-port models to describe the non-linear energy transfer. The idea is based on techniques similar to those applied for measuring linear acoustic two-port data for mufflers and other duct discontinuities ${ }^{21}$. It also has a theoretical basis in the framework for the polyharmonic distortion (PHD) model ${ }^{17-20}$ used for studying nonlinear properties for microwave systems.

\section{A. Polyharmonic distortion model}

In this section the theoretical foundations of the polyharmonic distortion (PHD) model will be introduced. The PHD approach assumes discrete tone or harmonic excitation and the problem we are solving can then be formulated as follows: determine the set of multivariate complex functions $F_{x n}$ that correlate all of the input pressure wave amplitudes $p_{A^{+}}(n f), p_{B^{+}}(n f)$ with the output pressure wave amplitudes $p_{A_{-}}(n f), p_{B_{-}}(n f)$. This is mathematically expressed as 


$$
\begin{aligned}
& p_{A-}(n f)=F_{A n}\left(p_{A+}(f), p_{B+}(f), p_{A+}(2 f), p_{B+}(2 f), p_{A+}(3 f), p_{B+}(3 f), \ldots \ldots \ldots\right) \\
& p_{B-}(n f)=F_{B n}\left(p_{A+}(f), p_{B+}(f), p_{A+}(2 f), p_{B+}(2 f), p_{A+}(3 f), p_{B+}(3 f), \ldots \ldots \ldots\right)
\end{aligned}
$$

for a system according to Figure 1 . The functions $F_{A n}, F_{B n}$ are called describing functions. The spectral mapping according to (15) is a general mathematical framework from which we can attempt to develop practical models in the frequency domain. The PHD model is a particular approximation, which involves the linearization using a set of assumptions. The first assumption is that the system studied is time-invariant. This implies that applying an arbitrary delay to the input signals in our case the incident $p_{A+}, p_{B^{+}}$waves, always results in exactly the same time delay for the output signals, the scattered $p_{A-}, p_{B-}$ waves. In the frequency domain, applying a time delay is equivalent to the application of a linear phase shift, which can be expressed as

$$
\begin{aligned}
& p_{A-}(n f) \cdot e^{j n \theta}=F_{A n}\left(p_{A+}(f) \cdot e^{j \theta}, p_{B+}(f) \cdot e^{j \theta}, p_{A+}(2 f) \cdot e^{j 2 \theta}, p_{B+}(2 f) \cdot e^{j 2 \theta},,, \ldots \ldots \ldots . .\right) \\
& p_{B-}(n f) \cdot e^{j n \theta}=F_{B n}\left(p_{A+}(f) \cdot e^{j \theta}, p_{B+}(f) \cdot e^{j \theta}, p_{A+}(2 f) \cdot e^{j 2 \theta}, p_{B+}(2 f) \cdot e^{j 2 \theta},,, \ldots \ldots \ldots . . .\right.
\end{aligned}
$$

The second assumption is that the functions $F_{A n}, F_{B n}$ are non-analytic because of the nonlinear system properties. Since (16) is valid for all values of $\theta$, we can make $\theta$ equal to the inverted phase of $p_{A_{+}}(f)$, the amplitude of the incident fundamental. We can now rewrite (16) as

$$
\begin{aligned}
& p_{A-}(n f) \cdot e^{-j n \phi\left(p_{A+}(f)\right)}=F_{A n}\left(\left|p_{A+}(f)\right|, p_{A+}(2 f) \cdot e^{-j 2 \phi\left(p_{A+}(f)\right)}, p_{B+}(2 f) \cdot e^{-j 2 \phi\left(p_{A+}(f)\right)}, \ldots \ldots \ldots . . .\right) \\
& \left.p_{B-}(n f) \cdot e^{-j n \phi\left(p_{A+}(f)\right)}=F_{B n}\left(\left|P_{A+}(f)\right|\right) \cdot p_{A+}(2 f) \cdot e^{-j 2 \phi\left(p_{A+}(f)\right)}, p_{B+}(2 f) \cdot e^{-j 2 \phi\left(p_{A+}(f)\right)}, \ldots \ldots \ldots . . .\right)
\end{aligned}
$$

In general, when the sample under test becomes nonlinear, the superposition principle is no longer valid. If it can be assumed that there is only one dominant large-signal input component present $\left(p_{A^{+}}(f)\right)$ whereas all other harmonic input components are relatively small, we will be able to use the superposition principle for the relatively small input components. This is called the harmonic superposition principle and is the key to the development of the PHD model. Linearization of (17) for all components besides the large signal $p_{A_{+}}(f)$ gives

$$
\begin{aligned}
& p_{A-}(n f)=F_{A n}\left(\mid p_{A+}(f), 0,0, \ldots \ldots \ldots . .\right) \cdot e^{j n \phi\left(p_{A+}(f)\right)}+Y_{A n A 2}\left(\mid p_{+}(f)\right) \cdot e^{j n \phi\left(p_{A+}(f)\right)} \cdot \operatorname{Re}\left(p_{A+}(2 f) \cdot e^{-j 2 \phi\left(p_{A+}(f)\right)}\right) \\
& \left.+\Psi_{A n A 2}\left(p_{A+}(f)\right)\right) \cdot e^{j n \phi\left(p_{A+}(f)\right)} \cdot \operatorname{Im}\left(p_{A+}(2 f) \cdot e^{-j 2 \phi\left(p_{A+}(f)\right)}\right)+Y_{A n B 2}\left(\left(p_{B+}(f)\right) \cdot e^{j n \phi\left(p_{A+}(f)\right)} \cdot \operatorname{Re}\left(p_{B+}(2 f) \cdot e^{-j 2 \phi\left(p_{A+}(f)\right)}\right)\right. \\
& +\Psi_{A n B 2}\left(\left(p_{A+}(f)\right)\right) \cdot e^{j n \phi\left(p_{A+}(f)\right)} \cdot \operatorname{Im}\left(p_{B+}(2 f) \cdot e^{-j 2 \phi\left(p_{A+}(f)\right)}\right)+\ldots \ldots \ldots \\
& p_{B-}(n f)=F_{B n}\left(\mid p_{A+}(f), 0,0, \ldots \ldots \ldots . .\right) \cdot e^{j n \phi\left(p_{A+}(f)\right)}+Y_{B n B 2}\left(\left(p_{+}(f)\right)\right) \cdot e^{j n \phi\left(p_{A+}(f)\right)} \cdot \operatorname{Re}\left(p_{B+}(2 f) \cdot e^{-j 2 \phi\left(p_{A+}(f)\right)}\right) \\
& \left.+\Psi_{B n B 2}\left(p_{A+}(f)\right)\right) \cdot e^{j n \phi\left(p_{A+}(f)\right)} \cdot \operatorname{Im}\left(p_{B+}(2 f) \cdot e^{-j 2 \phi\left(p_{A+}(f)\right)}\right)+Y_{B n B 2}\left(\left(p_{A+}(f)\right)\right) \cdot e^{j n \phi\left(p_{A+}(f)\right)} \cdot \operatorname{Re}\left(p_{B+}(2 f) \cdot e^{-j 2 \phi\left(p_{A+}(f)\right)}\right) \\
& +\Psi_{B n B 2}\left(\left(p_{A+}(f)\right)\right) \cdot e^{j n \phi\left(p_{A+}(f)\right)} \cdot \operatorname{Im}\left(p_{B+}(2 f) \cdot e^{-j 2 \phi\left(p_{A+}(f)\right)}\right)+\ldots \ldots \ldots .
\end{aligned}
$$

Note that the real and imaginary parts of the input arguments are treated as separate and independent variables because the spectral mapping function $F_{n}$ is assumed to be non-analytic. The final formulation of the PHD model equation is obtained by substituting the real and imaginary parts of the input amplitudes by a linear combination of the input amplitudes and their corresponding complex conjugates denoted by $p^{*}$. 


$$
\begin{aligned}
& p_{A-}(n f)=X_{A n A 1}\left(\left|p_{A+}(f)\right|\right) \cdot p_{A+}(f) \cdot e^{j(n-1) \phi\left(p_{A+}(f)\right)}+\sum_{i=2}^{\infty} X_{A n A i}\left(p_{A+}(f) \mid\right) \cdot p_{A+}(i f) \cdot e^{j(n-i) \phi\left(p_{A+}(f)\right)} \\
& +\sum_{i=2}^{\infty} \Xi_{A n A i}\left(\left|p_{A+}(f)\right|\right) \cdot p_{A+}^{*}(i f) \cdot e^{j(n+i) \phi\left(p_{A+}(f)\right)}+\sum_{i=1}^{\infty} X_{A n B i}\left(\left|p_{A+}(f)\right|\right) \cdot p_{B+}(i f) \cdot e^{j(n-i) \phi\left(p_{A+}(f)\right)} \\
& +\sum_{i=1}^{\infty} \Xi_{A n B i}\left(\left|p_{A+}(f)\right|\right) \cdot p_{B+}^{*}(i f) \cdot e^{j(n+i) \phi\left(p_{A+}(f)\right)} \\
& p_{B-}(n f)=\sum_{i=1}^{\infty} X_{B n A i}\left(\left|p_{A+}(f)\right|\right) \cdot p_{A+}(i f) \cdot e^{j(n-i) \phi\left(p_{A+}(f)\right)}+\sum_{i=2}^{\infty} \Xi_{B n A i}\left(\left|p_{A+}(f)\right|\right) \cdot p_{A+}^{*}(i f) \cdot e^{j(n+i) \phi\left(p_{A+}(f)\right)} \\
& +\sum_{i=1}^{\infty} X_{B n B i}\left(\left|p_{A+}(f)\right|\right) \cdot p_{B+}(i f) \cdot e^{j(n-i) \phi\left(p_{A+}(f)\right)}+\sum_{i=1}^{\infty} \Xi_{B n B i}\left(\mid p_{A+}(f)\right) \cdot p_{B+}^{*}(i f) \cdot e^{j(n+i) \phi\left(p_{A+}(f)\right)}
\end{aligned}
$$

If we assume that only the odd harmonics are important (19) can be rewritten as

$$
\begin{aligned}
& p_{A-}(n f)=X_{A n A 1}\left(\left|p_{A+}(f)\right|\right) \cdot p_{A+}(f) \cdot e^{j(n-1) \phi\left(p_{A+}(f)\right)} \\
& +\sum_{i=2}^{\infty} X_{A n A(2 i-1)}\left(\left|p_{A+}(f)\right|\right) \cdot p_{A+}((2 i-1) f) \cdot e^{j(n-(2 i-1)) \phi\left(p_{A+}(f)\right)} \\
& +\sum_{i=2}^{\infty} \Xi_{A n A(2 i-1)}\left(\left|p_{A+}(f)\right|\right) \cdot p_{A+}^{*}((2 i-1) f) \cdot e^{j(n+(2 i-1)) \phi\left(p_{A+}(f)\right)} . \\
& +\sum_{i=1}^{\infty} X_{A n B(2 i-1)}\left(\left|p_{A+}(f)\right|\right) \cdot p_{B+}((2 i-1) f) \cdot e^{j(n-(2 i-1)) \phi\left(p_{A+}(f)\right)} \\
& +\sum_{i=1}^{\infty} \Xi_{A n B(2 i-1)}\left(\mid p_{A+}(f)\right) \cdot p_{B+}^{*}((2 i-1) f) \cdot e^{j(n+(2 i-1)) \phi\left(p_{A+}(f)\right)}
\end{aligned}
$$

$$
\begin{aligned}
& p_{B-}(n f)=\sum_{i=1}^{\infty} X_{B n A(2 i-1)}\left(\left|p_{A+}(f)\right|\right) \cdot p_{A+}((2 i-1) f) \cdot e^{j(n-(2 i-1)) \phi\left(p_{A+}(f)\right)} \\
& +\sum_{i=2}^{\infty} \Xi_{B n A(2 i-1)}\left(\left(p_{A+}(f) \mid\right) \cdot p_{A+}^{*}((2 i-1) f) \cdot e^{j(n+(2 i-1)) \phi\left(p_{A+}(f)\right)}\right. \\
& +\sum_{i=1}^{\infty} X_{B n B(2 i-1)}\left(\left|p_{A+}(f)\right|\right) \cdot p_{B+}((2 i-1) f) \cdot e^{j(n-(2 i-1)) \phi\left(p_{A+}(f)\right)} \\
& +\sum_{i=1}^{\infty} \Xi_{B n B(2 i-1)}\left(\left(p_{A+}(f) \mid\right) \cdot p_{B+}^{*}((2 i-1) f) \cdot e^{j(n+(2 i-1)) \phi\left(p_{A+}(f)\right)}\right.
\end{aligned}
$$

The phase of the large signal input signal is here used as a reference for all harmonics. If we do not make the assumption that the describing function is non-analytic we get 


$$
\begin{aligned}
& p_{A-}(n f)=\sum_{i=1}^{\infty} X_{A n A(2 i-1)}\left(\left|p_{A+}(f)\right|\right) \cdot p_{A+}((2 i-1) f) \cdot e^{j(n-(2 i-1)) \phi\left(p_{A+}(f)\right)} \\
& +\sum_{i=1}^{\infty} X_{A n B(2 i-1)}\left(\left|p_{A+}(f)\right|\right) \cdot p_{B+}((2 i-1) f) \cdot e^{j(n-(2 i-1)) \phi\left(p_{A+}(f)\right)} . \\
& p_{B-}(n f)=\sum_{i=1}^{\infty} X_{B n A(2 i-1)}\left(\left|p_{A+}(f)\right|\right) \cdot p_{A+}((2 i-1) f) \cdot e^{j(n-(2 i-1)) \phi\left(p_{A+}(f)\right)} \\
& +\sum_{i=1}^{\infty} X_{B n B(2 i-1)}\left(\left|p_{A+}(f)\right|\right) \cdot p_{B+}((2 i-1) f) \cdot e^{j(n-(2 i-1)) \phi\left(p_{A+}(f)\right)}
\end{aligned}
$$

This is the same as the nonlinear scattering matrix suggested in Ref. 5 if the phase components, using the incident wave as phase reference, are included in the scattering matrix elements using the expression

$$
S_{n i}\left(\left|p_{+}(f)\right|\right)=X_{n i}\left(\left|p_{+}(f)\right|\right) e^{j(n-i) \phi\left(p_{+}(f)\right)} .
$$

This gives the nonlinear scattering matrix version of (21) as

$$
\begin{aligned}
& \left.p_{A-}(n f)=\sum_{i=1}^{\infty} S_{A n A(2 i-1)}\left(\mid p_{A+}(f)\right)\right) \cdot p_{A+}((2 i-1) f)+\sum_{i=1}^{\infty} S_{A n B(2 i-1)}\left(\left|p_{A+}(f)\right|\right) \cdot p_{B+}((2 i-1) f) \\
& \left.\left.p_{B-}(n f)=\sum_{i=1}^{\infty} S_{B n A(2 i-1)}\left(\mid p_{A+}(f)\right)\right) \cdot p_{A+}((2 i-1) f)+\sum_{i=1}^{\infty} S_{B n B(2 i-1)}\left(\mid p_{A+}(f)\right)\right) \cdot p_{B+}((2 i-1) f)
\end{aligned}
$$

\section{B. Single sided polyharmonic distortion model}

For a sample accessible only from one side, such as a complete liner sample, it is possible to develop single sided versions of the models described in the previous section. The single sided PHD model for nonlinear energy transfer to the higher order odd harmonics then becomes

$$
\begin{aligned}
& p_{A-}(n f)=X_{n 1}\left(\left|p_{A+}(f)\right|\right) \cdot p_{A+}(f) \cdot e^{j(n-1) \phi\left(p_{A+}(f)\right)} \\
& +\sum_{i=2}^{\infty} X_{n(2 i-1)}\left(\left|p_{A+}(f)\right|\right) \cdot p_{A+}((2 i-1) f) \cdot e^{j(n-(2 i-1)) \phi\left(p_{A+}(f)\right)}, \\
& +\sum_{i=2}^{\infty} \Xi_{n(2 i-1)}\left(\left|p_{A+}(f)\right|\right) \cdot p_{A+}^{*}((2 i-1) f) \cdot e^{j(n+(2 i-1)) \phi\left(p_{A+}(f)\right)}
\end{aligned}
$$

and the nonlineare scattering version will be

$$
p_{A-}(n f)=\sum_{i=1}^{\infty} S_{n(2 i-1)}\left(\left|p_{A+}(f)\right|\right) \cdot p_{A+}((2 i-1) f),
$$

where we have dropped the A-index in the system elements since we are only studying wave propagation on side A.

\section{Energy considerations}

6

American Institute of Aeronautics and Astronautics 
One interesting question which can be answered using the techniques discussed in this paper is what happens to the energy. Once we have performed the two-microphone wave decomposition we can calculate the power of the waves propagating in the positive and negative x-direction, as described in equations (11) to (14), at the excitation frequency and at the higher harmonics. If we just look at the excitation frequency energy conservation gives

$$
W_{A+}(f)+W_{B+}(f)=W_{A-}(f)+W_{B-}(f)+W_{a b s}(f)
$$

where $W_{a b s}(f)$ is the power dissipated at the excitation frequency which partly is lost into heat and vorticity but partly is radiated as acoustic power at higher harmonics. It is now possible to define a reflection factor $(r(f))$ a transmission factor $(\tau(f))$ and an absorption factor $(\alpha(f))$ as

$$
\begin{gathered}
r(f)=\frac{W_{A-}(f)}{W_{A+}(f)}, \\
\tau(f)=\frac{W_{B-}(f)-W_{B+}(f)}{W_{A+}(f)}, \\
\alpha(f)=\frac{W_{A+}(f)-W_{A-}(f)+W_{B+}(f)-W_{B-}(f)}{W_{A+}(f)},
\end{gathered}
$$

where

$$
r(f)+\tau(f)+\alpha(f)=1
$$

because of energy conservation. This can be extended to take the energy at the higher harmonics into account which gives

$$
\sum_{i=1}^{\infty} W_{A+}((2 i-1) f)+W_{B+}((2 i-1) f)=\sum_{i=1}^{\infty} W_{A-}((2 i-1) f)+W_{B-}((2 i-1) f)+W_{a b s T}
$$

where $W_{a b s T}$ is the power dissipated into heat and vorticity. We can now define an additional reflection factor $(r(n f))$ and transmission factor $(\tau(n f))$ at each harmonic as

$$
\begin{aligned}
& r(n f)=\frac{W_{a-}(n f)-W_{a+}(n f)}{W_{a+}(f)}, \\
& \tau(n f)=\frac{W_{b-}(n f)-W_{b+}(n f)}{W_{a+}(f)},
\end{aligned}
$$

where $r(n f)$ and $\tau(n f)$ are interpreted as the fraction of acoustic power generated on each side of the sample at frequency $\mathrm{n} f$. The total absorption factor is given by

$$
\alpha_{T}=\frac{\sum_{i=1}^{\infty} W_{A+}((2 i-1) f)+W_{B+}((2 i-1) f)-\sum_{i=1}^{\infty} W_{A-}((2 i-1) f)+W_{B-}((2 i-1) f)}{W_{A+}(f)},
$$

where now 


$$
\sum_{i=1}^{\infty}(r((2 i-1) f)+\tau((2 i-1) f))+\alpha_{T}=1
$$

It is to be noted that all of these expressions depend on the downstream, and upstream, termination and not only on the properties of the sample. Using the two-port equations defined in section III A we can get a result for a nonreflecting termination on both the A and B-side of the sample which only depends on the properties of the sample. If the duct termination on both sides of the sample is reflection free all the $p_{A+}(n f)$ and $p_{B+}(n f)$ except $p_{A+}(f)$ are equal to zero and, using the nonlinear scattering matrix formulation of equation (23), we get $p_{A_{-}}(n f)=S_{A n A 1}\left(\left|p_{A_{+}}(f)\right|\right) p_{A_{+}}(f)$ and $p_{B-}(n f)=S_{B n A 1}\left(\left|p_{A_{+}}(f)\right|\right) p_{A_{+}}(f)$ which gives the expressions

$$
\begin{gathered}
r(n f)=\frac{W_{a-}(n f)}{W_{a+}(n f)}=\left|S_{A n A 1}\left(\left|p_{A+}(f)\right|\right)\right|^{2}, \\
\tau(n f)=\frac{W_{b-}(n f)}{W_{a+}(n f)}=\mid S_{B n A 1}\left(\left.\left|p_{A+}(f)\right|\right|^{2},\right. \\
\alpha_{T}=1-\sum_{i=1}^{\infty} \mid S_{A(2 i-1) A 1}\left(\left|p_{A+}(f)\right|||^{2}-\sum_{i=1}^{\infty} \mid S_{B(2 i-1) A 1}\left(\left.\left|p_{A+}(f)\right|\right|^{2} .\right.\right.
\end{gathered}
$$

\section{Determination of multi-port data}

\section{A. Application of multi-load techniques}

In the linear case the traditional scattering matrix two-port relationship between side A and B of the sample shown in Fig. 1 gives a relation between the pressure wave amplitudes on each side of the sample on a frequency by frequency basis. This system of equations has four unknowns and two equations so we need measurement for at least two independent acoustic test cases to solve it. This can be achieved by using different terminations (loads) on the B-side or by exciting with sources on the A and B-sides ${ }^{21}$. If we now consider the two-sided nonlinear multiport equation systems given in equations (20) and (23) and take $N$ harmonics into account, we have $2 N$ equations. Equation (23) have $(2 N)^{2}$ unknowns so $2 N$ acoustic test states would be needed for solving for the unknown quantities. In the PHD model of equation (20) we have $2 N \mathrm{x}(4 N-1)$ unknowns so that $4 N-1$ acoustic test cases would be needed. For the one-sided versions in equations (24) and (25) we have $\mathrm{N}$ equations and $2 N x(4 N-1)$ and $N^{2}$ unknowns. Since we need to control the magnitude of the incident pressure wave on the A-side so that it is the same for all acoustic test cases a multi-load technique where the loading on the B-side has been varied was selected for the two-sided multi-ports and a variation in the A-side loading was used for the single-sided two-ports. The number of acoustic loads grows quickly with the number of harmonics studied so some simplifications to reduce the number of required loads are of interest. The main simplification used is the assumption that the non-linear energy transfer only takes place in the direction from lower frequencies to higher harmonics. Experimental evidence shows that we generate odd higher order harmonics $(n=3,5,7, \ldots .$.$) but no sub-harmonics. In this case a number of terms in$ equations (20)-(25) would be equal to zero leading to for the two-sided PHD model 


$$
\begin{aligned}
& p_{A-}(n f)=X_{A n A 1}\left(\left|p_{A+}(f)\right|\right) \cdot p_{A+}(f) \cdot e^{j(n-1) \phi\left(p_{A+}(f)\right)}+ \\
& +X_{A n B 1}\left(\left|p_{A+}(f)\right|\right) \cdot p_{B+}(f) \cdot e^{j(n-1) \phi\left(p_{A+}(f)\right)}+\Xi_{A n B 1}\left(\left|p_{A+}(f)\right|\right) \cdot p_{B+}^{*}(f) \cdot e^{j(n+1) \phi\left(p_{A+}(f)\right)} \\
& +X_{A n A n}\left(\left|p_{A+}(f)\right|\right) \cdot p_{A+}(n f)+\Xi_{A n A n}\left(\left|p_{A+}(f)\right|\right) \cdot p_{A+}^{*}(n f) \cdot e^{j 2 n \phi\left(p_{A+}(f)\right)} \\
& +X_{A n B n}\left(\left|p_{A+}(f)\right|\right) \cdot p_{B+}(n f)+\Xi_{A n B n}\left(\left|p_{A+}(f)\right|\right) \cdot p_{B+}^{*}(n f) \cdot e^{j 2 n \phi\left(p_{A+}(f)\right)}
\end{aligned}
$$

$$
\begin{aligned}
& p_{B-}(n f)=X_{B n A 1}\left(\left|p_{A+}(f)\right|\right) \cdot p_{A+}(f) \cdot e^{j(n-1) \phi\left(p_{A+}(f)\right)}+ \\
& +X_{B n B 1}\left(\left|p_{A+}(f)\right|\right) \cdot p_{B+}(f) \cdot e^{j(n-1) \phi\left(p_{A+}(f)\right)}+\Xi_{B n B 1}\left(\left|p_{A+}(f)\right|\right) \cdot p_{B+}^{*}(f) \cdot e^{j(n+1) \phi\left(p_{A+}(f)\right)}, \\
& \left.+X_{B n A n}\left(\mid p_{A+}(f)\right)\right) \cdot p_{A+}(n f)+\Xi_{B n A n}\left(\left|p_{A+}(f)\right|\right) \cdot p_{A+}^{*}(n f) \cdot e^{j 2 n \phi\left(p_{A+}(f)\right)} \\
& \left.+X_{B n B n}\left(\mid p_{A+}(f)\right)\right) \cdot p_{B+}(n f)+\Xi_{B n B n}\left(\left|p_{A+}(f)\right|\right) \cdot p_{B+}^{*}(n f) \cdot e^{j 2 n \phi\left(p_{A+}(f)\right)},
\end{aligned}
$$

and for the two-sided nonlinear scattering matrix

$$
\begin{aligned}
& p_{A-}(n f)=S_{A n A 1}\left(\left|p_{A+}(f)\right|\right) \cdot p_{A+}(f)+S_{A n B 1}\left(\left|p_{A+}(f)\right|\right) \cdot p_{B+}(f) \\
& +S_{A n A n}\left(\left|p_{A+}(f)\right|\right) \cdot p_{A+}(n f)+S_{A n B n}\left(\left|p_{A+}(f)\right|\right) \cdot p_{B+}(n f) \\
& p_{B-}(n f)=S_{B n A 1}\left(\left|p_{A+}(f)\right|\right) \cdot p_{A+}(f)+S_{B n B 1}\left(\left|p_{A+}(f)\right|\right) \cdot p_{B+}(f) \\
& +S_{B n A n}\left(\left|p_{A+}(f)\right|\right) \cdot p_{A+}(n f)+S_{B n B n}\left(\left|p_{A+}(f)\right|\right) \cdot p_{B+}(n f)
\end{aligned}
$$

which means that we need seven loads to solve equation (39) and four loads to solve equation (40). For the onesided PHD model we get

$$
\begin{aligned}
& \left.p_{A-}(n f)=X_{n 1}\left(\mid p_{A+}(f)\right)\right) \cdot p_{A+}(f) \cdot e^{j(n-1) \phi\left(p_{A+}(f)\right)}+ \\
& +X_{n n}\left(\left|p_{A+}(f)\right|\right) \cdot p_{A+}(n f)+\Xi_{n n}\left(\left|p_{A+}(f)\right|\right) \cdot p_{A+}^{*}(n f) \cdot e^{j 2 n \phi\left(p_{A+}(f)\right)},
\end{aligned}
$$

and for the one-sided nonlinear scattering matrix

$$
p_{A-}(n f)=S_{n 1}\left(\left|p_{A+}(f)\right|\right) \cdot p_{A+}(f)+S_{n n}\left(\left|p_{A+}(f)\right|\right) \cdot p_{A+}(n f),
$$

which means that we need three loads to solve equation (41) and two loads to solve equation (42).

One further assumption which can be made is that the level of excitation at $3 f$ and higher harmonics is low and that we are in the linear regime at these frequencies. Then the nonlinear scattering matrix terms $S_{A n A n}, S_{A n B n}, S_{B n A n}$ and $S_{B n B n}$ can be determined by a separate measurement using low level excitation at frequency $n f$. The validity of this assumption can be checked by comparing the level at $n f$ when the system is excited at frequency $f$ with the level used for the test with excitation at $n f$. Equation (23) can then be divided into three separate equations

$$
\begin{aligned}
& p_{A-}(f)=S_{A 1 A 1}\left(\left|p_{A+}(f)\right|\right) \cdot p_{A+}(f)+S_{A 1 B 1}\left(\left|p_{A+}(f)\right|\right) \cdot p_{B+}(f) \\
& p_{B-}(f)=S_{B 1 A 1}\left(\left|p_{A+}(f)\right|\right) \cdot p_{A+}(f)+S_{B 1 B 1}\left(\left|p_{A+}(f)\right|\right) \cdot p_{B+}(f)
\end{aligned},
$$




$$
\begin{aligned}
& p_{A-}(n f)-S_{A n A n}\left(\left|p_{A+}(f)\right|\right) \cdot p_{A+}(n f)-S_{A n B n}\left(\left|p_{A+}(f)\right|\right) \cdot p_{B+}(n f)= \\
& S_{A n A 1}\left(\left|p_{A+}(f)\right|\right) \cdot p_{A+}(f)+S_{A n B 1}\left(\left|p_{A+}(f)\right|\right) \cdot p_{B+}(f) \\
& p_{B-}(n f)-S_{B n A n}\left(\left|p_{A+}(f)\right|\right) \cdot p_{A+}(n f)-S_{B n B n}\left(\left|p_{A+}(f)\right|\right) \cdot p_{B+}(n f)= \\
& S_{B n A 1}\left(\left|p_{A+}(f)\right|\right) \cdot p_{A+}(f)+S_{B n B 1}\left(\left|p_{A+}(f)\right|\right) \cdot p_{B+}(f) \\
& p_{A-}(n f)=S_{A n A n}\left(\left|p_{A+}(f)\right|\right) \cdot p_{A+}(n f)+S_{A n B n}\left(\left|p_{A+}(f)\right|\right) \cdot p_{B+}(n f) \\
& p_{B-}(n f)=S_{B n A n}\left(\left|p_{A+}(f)\right|\right) \cdot p_{A+}(n f)+S_{B n B n}\left(\left|p_{A+}(f)\right|\right) \cdot p_{B+}(n f)
\end{aligned}
$$

Here we obviously need two independent acoustic test cases to solve for the unknowns.

For the one-sided nonlinear scattering matrix $S_{n n}$ can using this assumption be measured by a separate low level reflection coefficient measurement. We then only need one acoustic test case to determine the remaining $S_{n 1}$ term.

\section{B. Alignment of input data}

One problem with the multi-load loads techniques used here is that the level of excitation at the fundamental frequency needs to be the same for each acoustic load used. A total of 9 different loads consisting of open and closed pipes of different lengths were used for the two-sided multi-port experiments. For the one-sided tests five loads on the A-side were used. To get the same non-linear forcing at the sample for different acoustic loads it was attempted to keep the magnitude of the incident pressure wave on the A-side of the sample constant. For the first sample tested a number of different excitation levels were set by varying the input level to the loudspeaker. For the subsequent acoustic loads it was attempted to set the magnitude of the incident pressure to the same as for the first load by testing with a number of different input level settings until a reasonable agreement was obtained. This was attempted but was not completely successful. Since a variation in the excitation levels used to solve for the nonlinear two-port matrix components would be a source of error in the result a method to align the excitation levels for the different acoustic loads with each other was developed. This method is based on the assumption that the travelling wave components at the frequencies of interest depend slowly on the amplitude of the fundamental frequency so that a fit of the experimental data to a common set of excitation amplitudes can be made. Two different methods were tested: a polynomial fit and a cubic spline fit. For the polynomial fit the polynomial order was first increased until an optimum agreement was obtained between the model and the real and imaginary part of the experimental data points for the travelling wave components. This turned out to produce spurious variations in the final results since also variations due to experimental error was modelled. In the end the order of the polynomials were reduced to give a more reasonable result. The polynomial order used was between 2 and 5 for the travelling wave components studied. The cubic spline fit had the same problem as the high order polynomial fit in that it introduced spurious oscillations in the results. Figure 2 shows some examples of the polynomial fits used compared to the experimental data. 


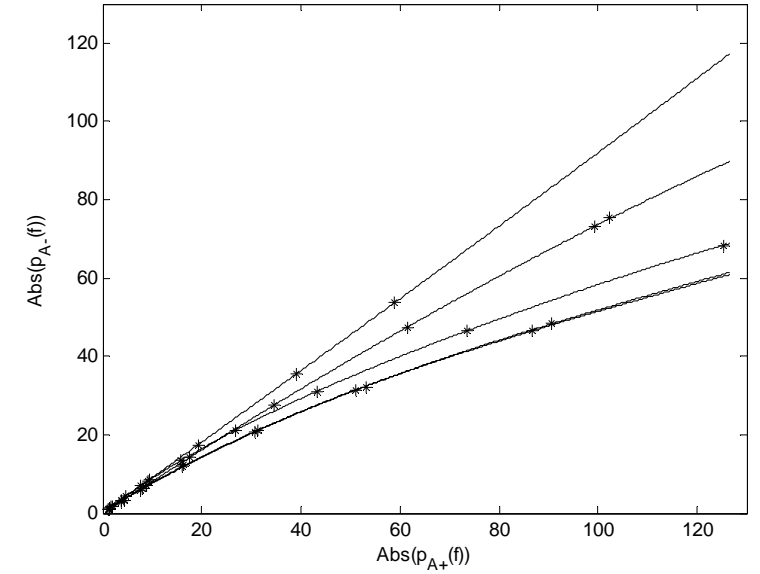

(a)

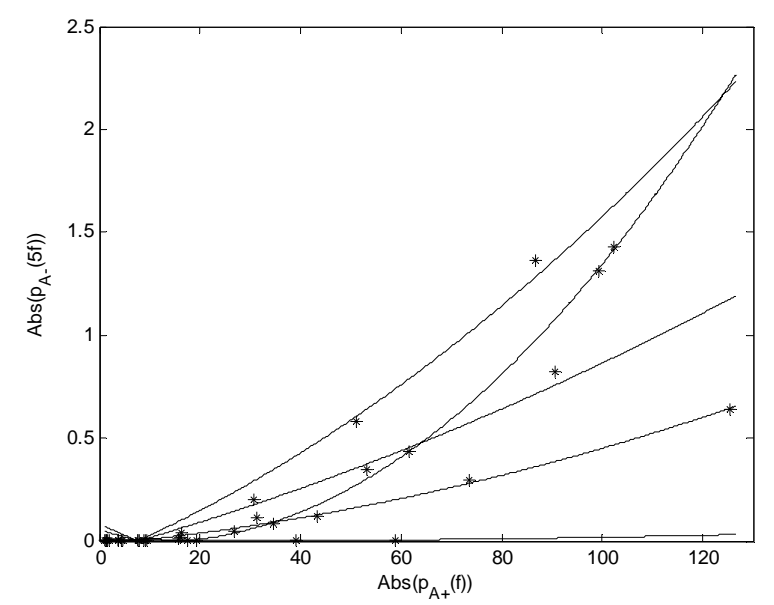

(c)

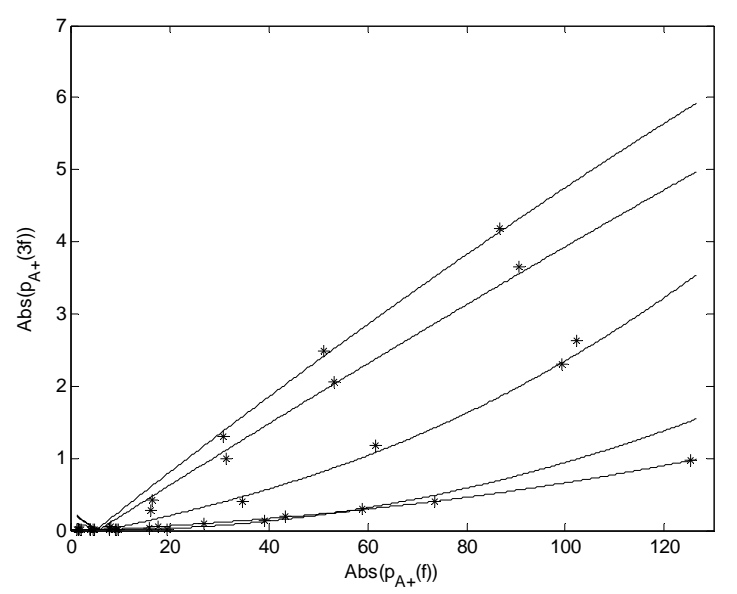

(b)

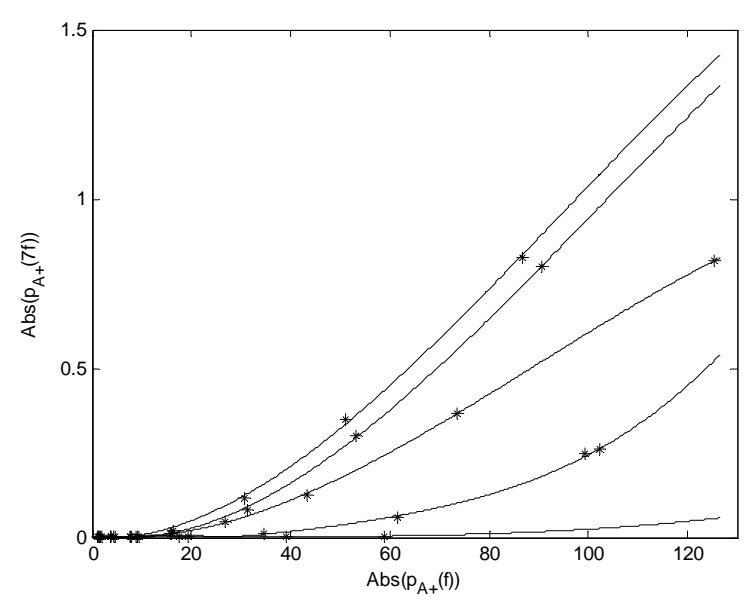

(d)

Figure 2. Magnitude of travelling wave components as a function of $\left|p_{+}(f)\right|$ : full lines - polynomial fit, stars experimental data, (a) $\left|p_{B-}(f)\right|$ polynomial order 3, (b) $\left|p_{A_{-}}(3 f)\right|$ polynomial order 2, (c) $\left|p_{B_{-}}(5 f)\right|$ polynomial order 2 , (d) $\left|p_{A+}(7 f)\right|$ polynomial order 3 .

\section{Over-determination and linearity tests}

In section IV A it was discussed how many acoustic test cases (loads) that are needed to solve for the nonlinear multi-port data. The number varied between one and seven depending on the complexity of the model and the approximations made. In the experimental tests performed for this study measurements were made for nine different acoustic loads for the two-sided two-port experiments and five loads for the one-sided two-port experiments, which give the possibility for some over-determination. This can be used to improve the quality of the results but also to assess how well the data fits the linear models used. The formulation we are using gives a matrix relation between the vectors of the travelling wave components reflected or radiated from the sample (p.) and incident on the sample $\left(\mathbf{p}_{+}\right)$of the type $\mathbf{p}_{-}=\mathbf{A} \mathbf{p}_{+}$. The elements of matrix $\mathbf{A}$ are obtained by matrix inversion $A=\mathbf{p}_{-} \cdot \mathbf{p}_{+}^{-1}$. This can be done for each row of the matrix separately. With over-determination it is possible to formulate a linearity test in analogy with what has been introduced for acoustic source characterisation ${ }^{24}$ 


$$
\gamma=\mathbf{p}_{-} \cdot \mathbf{p}_{+}^{-1} \cdot \mathbf{p}_{+} \cdot \mathbf{p}_{-}^{-1}
$$

If this is performed for each row of the matrix expression separately and using $N$ loads $\mathbf{p}$. will be of order $1 \mathrm{x} N$, and $\mathbf{p}_{+}$will be of the order $N \mathrm{x} N$ giving $\gamma$ of the order $1 \mathrm{x} 1$, that is one value for the linearity test for each row corresponding to an component in $\mathbf{p}$. This linearity test will tell us how well the experimental data fits the linear model.

Another way to assess the outcome of the system identification is to calculate a residual defined as

$$
\text { Residual }=\sqrt{\left(\mathbf{p}_{-}-\mathbf{A} \cdot \mathbf{p}_{+}\right)^{-1} \cdot\left(\mathbf{p}_{-}-\mathbf{A} \cdot \mathbf{p}_{+}\right)} .
$$

To make this non-dimensional we can divide by $\mathbf{p}$ - giving

$$
\text { Residual }=\sqrt{\left(\left(\mathbf{p}_{-}-\mathbf{A} \cdot \mathbf{p}_{+}\right) / \mathbf{p}_{-}\right)^{-1} \cdot\left(\left(\mathbf{p}_{-}-\mathbf{A} \cdot \mathbf{p}_{+}\right) / \mathbf{p}_{-}\right)},
$$

which, similarly to the linearity test, will give an indication of how well the experimental data fitted the model

\section{Error analysis}

It is important to obtain an estimate of the errors in the experimental data to be able to get an idea about the errors in the final result. Since the measurements made for this study were made in close to ideal conditions in an impedance tube with low background level and sinusoidal excitation it is to be expected that the errors in the measured pressures is small and in the order of magnitude $1 \%$. The microphone separation used for the twomicrophone wave decomposition ${ }^{21-22}$ was $0.1 \mathrm{~m}$. Using the analysis of the sensitivity of the two-microphone wave decomposition to errors in the input data ${ }^{25-26}$ it is possible estimate the risk for an increase in experimental error in the measured travelling wave components. Within the Helmholtz number range

$$
0.1 \pi \leq k s \leq 0.8 \pi \text {. }
$$

the error will not be increased with more than a factor 10. The factor 10 applies at the upper and lower end of the range where $k s \approx 0.1 \pi$ or $k s \approx 0.8 \pi$, while there is no error increase around $k s \approx 0.5 \pi$. The range given in (50) is in fact the first Helmholtz number range and can be extended to higher frequencies through

$$
1.1 \pi \leq k s \leq 1.8 \pi,
$$

where we now have a risk for error amplification by a factor 10 around $k s \approx 1.1 \pi$ and $k s \approx 1.8 \pi$ while the risk for error is low around $k s \approx 1.5 \pi$. In the single tone excitation experiments presented here the fundamental excitation frequency was around $210 \mathrm{~Hz}$. The other odd harmonics of interest are then $630 \mathrm{~Hz}, 1050 \mathrm{~Hz}, 1470 \mathrm{~Hz}$ and $1890 \mathrm{~Hz}$. With $\mathrm{s}=0.1 \mathrm{~m}$ these frequencies give Helmholtz numbers: $0.12 \pi, 0.37 \pi, 0.61 \pi, 0.86 \pi$ and $1.10 \pi$. This means that there is a low risk for error amplification at $630 \mathrm{~Hz}$ and $1050 \mathrm{~Hz}$, while there is a risk for some amplification of error at $210 \mathrm{~Hz}$ and $1890 \mathrm{~Hz}$. At $1470 \mathrm{~Hz}$ we are slightly outside of the range given in (50) and there is a risk for an amplification of errors by at least a factor of 10. Results will never the less be given for this frequencies in some cases, but the risk for larger errors should in this case be kept in mind.

The sensitivity of the calculated two-port results to these errors can be estimated in different ways. One possibility is to perform a numerical simulation of the effect of errors in the input data. Some results of this type of simulation will be provided in section V B. It is obviously also possible to calculate a condition number for the inversion of $\mathbf{p}_{+}$giving the ratio between the largest and smallest singular value. A large condition number indicates that the inversion is ill-conditioned. Typically the inverse of the condition number should not be smaller than the error in the $\mathbf{p}_{+}$components. This means that for a condition number larger than 100 there is a risk that an error larger $1 \%$ will be amplified in the final scattering matrix results. The matrix inversion has been performed using Matlab pseudo-inverse which is based on singular value decomposition. Here it is possible to set a tolerance so that singular values smaller than the tolerance are treated as zero. This has been used to regularize the matrix inversion. 


\section{Experimental results and discussion}

\section{A. Test setup}

Tests of the techniques described in sections III and IV have been made using a duct as shown in Figure 1, where the sample was mounted in the centre with microphones on each side and a loudspeaker at one end. The microphone separation was $s=0.1 \mathrm{~m}$ and the distance between the sample and the first microphones on each side was $L=0.225$ $\mathrm{m}$. The sample studied was a perforate with circular holes with $1 \mathrm{~mm}$ diameter, $2 \mathrm{~mm}$ plate thickness and $2 \%$ percentage open area. The acoustic loads at the downstream (B) side of the sample or the upstream (A) side were varied, by applying open and closed pipes of varying lengths, in order to create different acoustic test states.

\section{B. Preliminary investigations}

In Figure 3 the variation in sound pressure level, for a specific load on the B-side, as the level of the single frequency excitation at $210 \mathrm{~Hz}$ is increased is shown. Figure 4 shows the variation in corresponding particle velocities. The pressure difference over the sample can be clearly seen while the particle velocity is the same at both sides of the sample. The levels at the fundamental frequency are much higher than at the higher harmonics. It could be expected that the level at $3 f$ would be the second in magnitude, but the levels depend on the reflections from the terminations on the A and B sides of the sample.

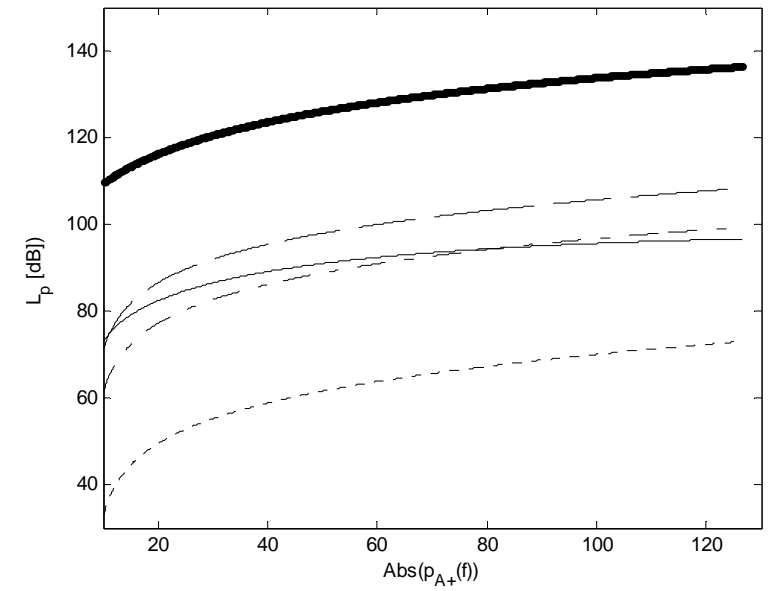

(a)

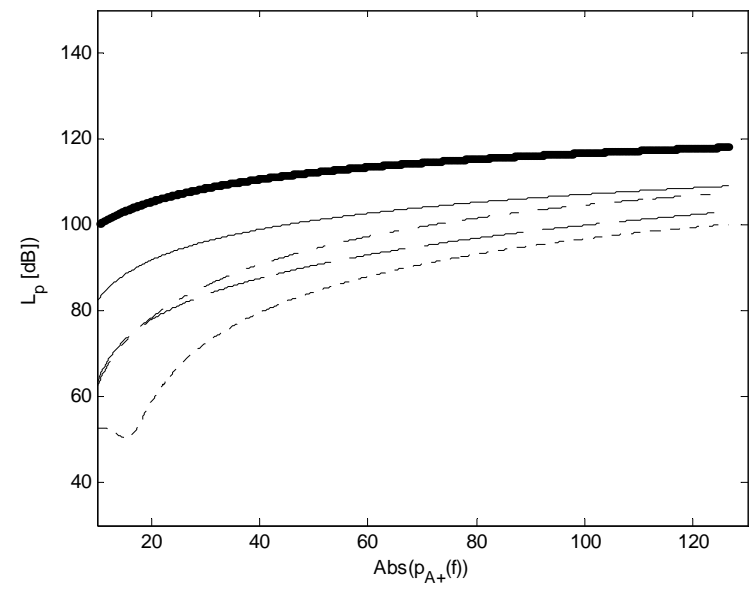

(b)

Figure 3. Variation in sound pressure level (re. $210^{-5} \mathrm{~Pa}$ ) with magnitude of the incident pressure wave as the level of the single frequency excitation at $210 \mathrm{~Hz}$ is increased for one of the test cases used in the present study: thick solid line $-210 \mathrm{~Hz}$, solid line $-630 \mathrm{~Hz}$, dashed line $-1050 \mathrm{~Hz}$, dashed dotted line $-1470 \mathrm{~Hz}$, dotted line - 1890 Hz. (a) A-side, (b) B-side. 


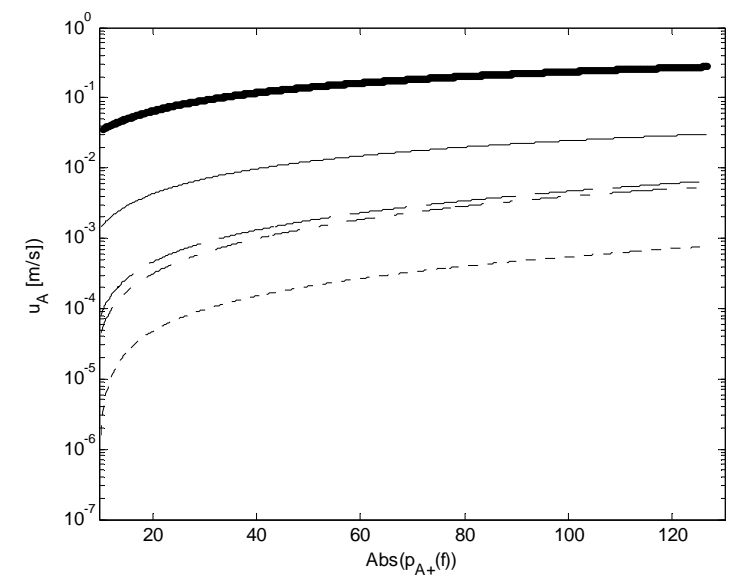

(a)

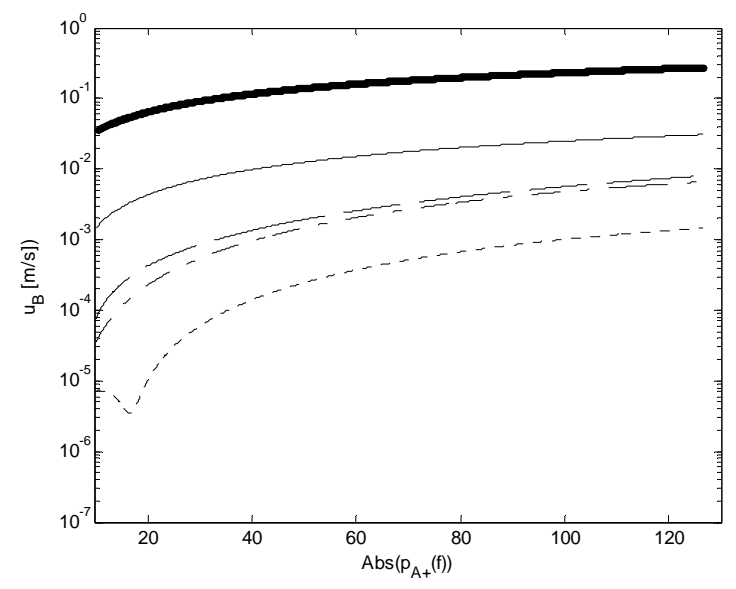

(b)

Figure 4. Variation in particle velocity with magnitude of the incident pressure wave as the level of the single frequency excitation at $210 \mathrm{~Hz}$ is increased for one of the test cases used in the present study: thick solid line $210 \mathrm{~Hz}$, solid line $-630 \mathrm{~Hz}$, dashed line $-1050 \mathrm{~Hz}$, dashed dotted line $-1470 \mathrm{~Hz}$, dotted line - $1890 \mathrm{~Hz}$. (a) Aside, (b) B-side.

\section{Error analysis and linearity tests}

Solving the equations to get the PHD matrix or nonlinear scattering matrix elements involves the inversion of a matrix. The conditioning of this matrix will determine how sensitive the result is to errors in the input data. As discussed in section IV D a condition number can be calculated for the matrix to be inverted and this information can be used to assess the sensitivity to errors. The matrix inversion is performed using Matlab pseudo-inverse which uses singular value decomposition. The condition number gives the ratio between the largest and smallest singular value. The interpretation is that a for a condition number larger than $C$ a relative error in the input data larger than $1 / C$ will be amplified by the matrix inversion. The pseudo-inverse function can introduce a tolerance value meaning that any singular value smaller than the tolerance will be treated as zero. This will regularize the matrix inversion and reduce the sensitivity to errors in the input data. The information can also be used to calculate the matrix rank for a given tolerance giving an estimate of the number of independent equations (rows), which is also the same as the number of singular values larger than the tolerance. Figure 5 shows condition numbers for the calculation of the two-sided nonlinear scattering matrix according to equation (40) and according to the two-sided PHD model in equation (39) as function of the magnitude of the incident pressure wave. It can be seen that for the excitation frequency component $f$ the condition number is smaller than 10 indicating a low sensitivity to errors in the input data. For the higher harmonics the condition number is between 200 and 13000. In all these cases it is to be expected that there will an amplification of errors in the input data unless regularization of the matrix inversion is applied. 


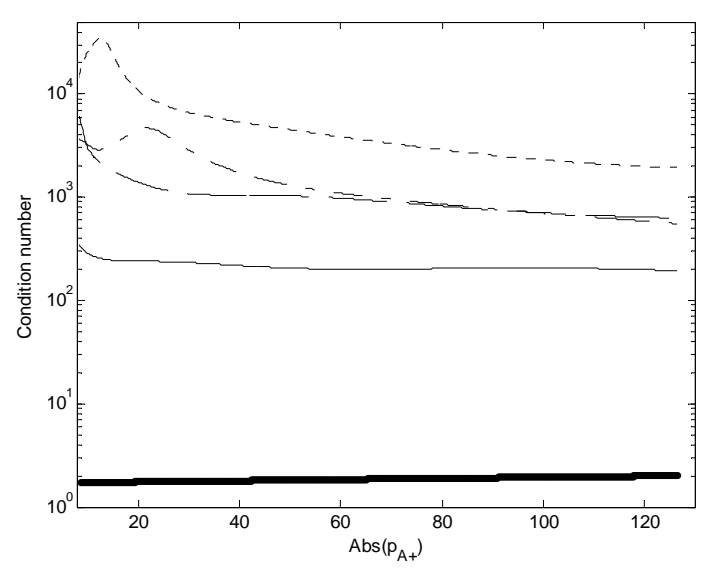

(a)

Figure 5. Condition numbers as function of the magnitude of the incident pressure wave: solid line $-f$, dashed line - 3f, dashed dotted line $-5 f$, dotted line - $9 f$. (a) Two-sided nonlinear scattering matrix according to equation (41), (b) Two-sided PHD model according to equation (39).

For the one-sided nonlinear two-port experiments the condition numbers for the matrix inversions are shown in Figure 6. It can be seen that the condition number is smaller than 100 for the $3 f$ component except perhaps for the highest excitation levels for the PHD model. For the $5 f$ component the condition number is between 100 and 300 . The $7 f$ component has a fairly high risk for error amplification especially for the PHD model with condition numbers between 500 and 2000. The risk for error amplification is high for all levels of excitation for the $9 f$ component. Matrix regularization has been applied for the $5 f, 7 f$ and $9 f$ components when calculating the PHD model data reducing the matrix rank from 3 (three unknowns) to 2.

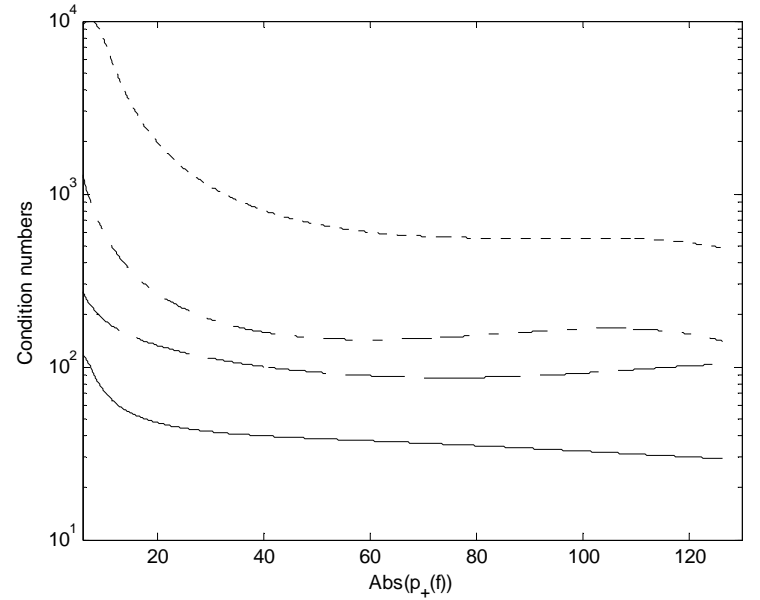

(a)

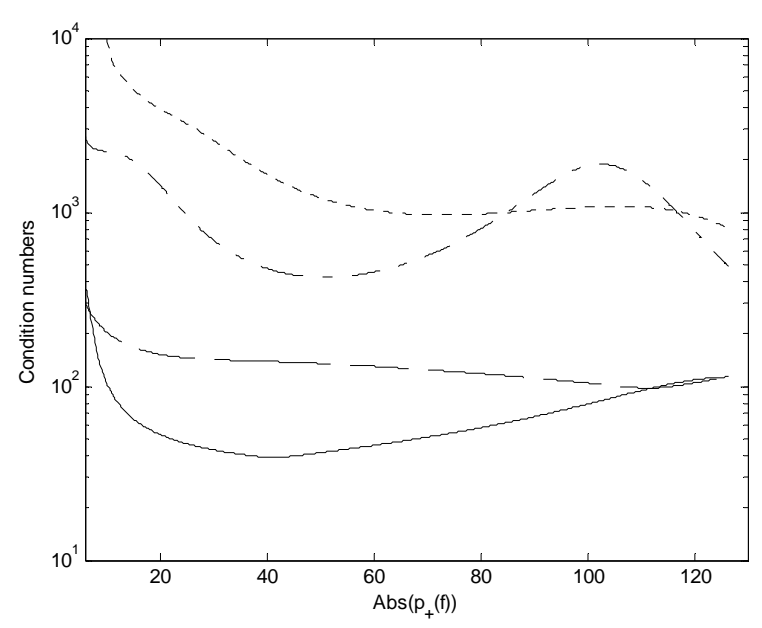

(b)

Figure 6. Condition numbers for the matrix inversions performed to calculate the different model results as function of the magnitude of the incident pressure wave: solid line - results at $3 f$, dashed line - results at $5 f$, dashed-dotted line - results at $7 f$, dotted line - results at $9 f$. (a) One-sided scattering matrix model according to (42), (b) One-sided PHD model according to (41).

The effect of errors during the calculation of multi-port data has also been investigated by numerical simulation. The error has been introduced as a relative error in the magnitude of travelling pressure components for 50 randomly distributed phase angles. This has made it possible to estimate standard deviations and relative errors in the calculated quantities. Figure 7 shows some examples of the results, for the estimate of the reflection factor according to equation (32), for the case with reflection free terminations. The result without regularization in Figure 7 (a) gives large amplification of the error in input data. When regularisation is applied by assuming a relative error of $1 \%$ 
simulation results and also applying 1\% error in the input data, Figure 7 (b) shows that the resulting simulated error is also $1 \%$. When the error in the input data is increased to $10 \%$ the resulting error increases to $10 \%$ and when the error used for matrix regularisation is increased to $10 \%$ giving a reduction in matrix rank a simulation using a $1 \%$ relative error in the input data indeed gives a relative error around $1 \%$. These results gives confidence in the assumption that applying the regularization techniques will make it possible to obtain results without amplification of errors in the input data.

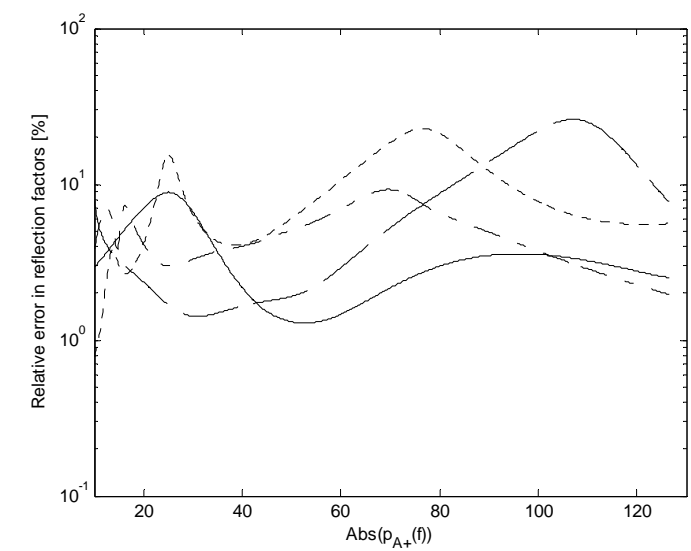

(a)

Figure 7. Simulated relative errors in the calculated reflection factor according to equation (34) as function of magnitude of the incident pressure wave: solid line $-3 f$, dashed line $-5 f$, dashed dotted line $-7 f$, dotted line 9f. (a) No matrix regularization applied, $1 \%$ error simulated in input data, (b) matrix regularization applied assuming $1 \%$ error in the input data, $1 \%$ error simulated in input data.

Linearity tests according to equation (46) have been calculated and Figure 8 shows results for the nonlinear scattering matrix according to equation (40) and the PHD matrix according to equation (39) for different assumed levels or errors in the input data. It can be seen that for the excitation frequency $(f)$ there is always a perfect linear fit. For the $3 f$ component the result is also good for $0 \%$ and $1 \%$ error in the input data while it reduces to around 0.7 0.8 for $10 \%$ assumed error in the input data. For the $5 f$ component the results reduce to around $0.7-0.8$ for $1 \%$ and $10 \%$ assumed input error. The higher harmonics ( $7 f$ and $9 f$ ) show a more scattered result with lower values.

Remember that it is expected that the error for $7 f$ is higher since it is outside the frequency region where the twomicrophone wave decomposition does not give a large increase in errors in experimental input data.

The conclusion from these preliminary investigations is that there is a need for matrix regularization. The results presented in the remaining sections are all based on assuming a 1\% error in input data. It is to be expected that the results at the excitation frequency $f$ are highly reliable. For the $3 f$ and $5 f$ components the results are expected to be fairly reliable while they should be less reliable at $7 f$ and $9 f$. 


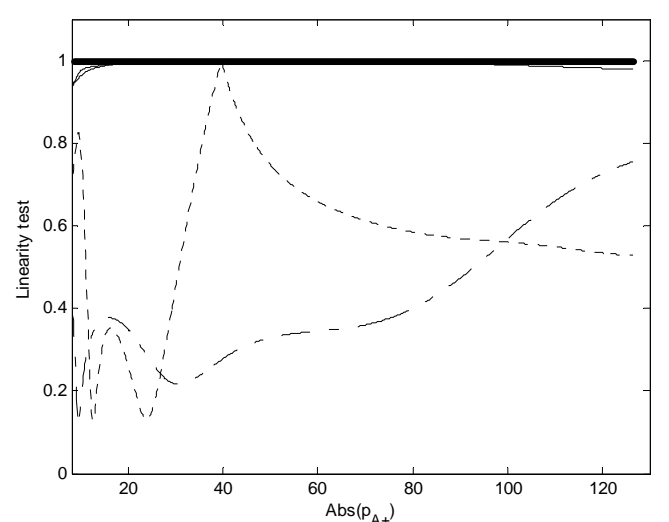

(a)

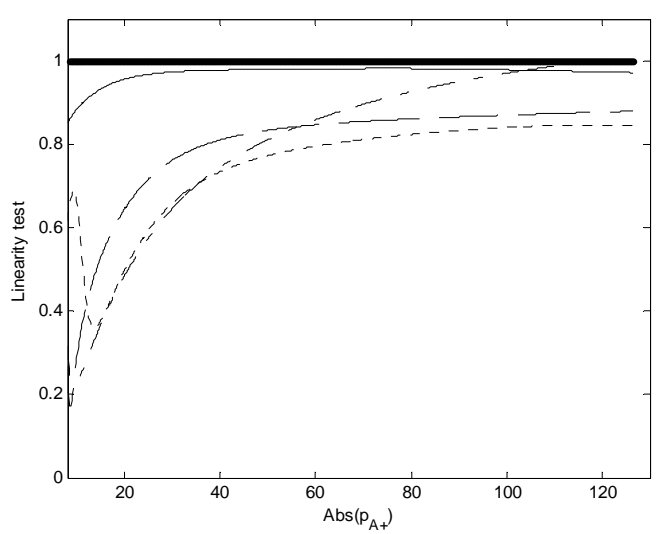

(c)

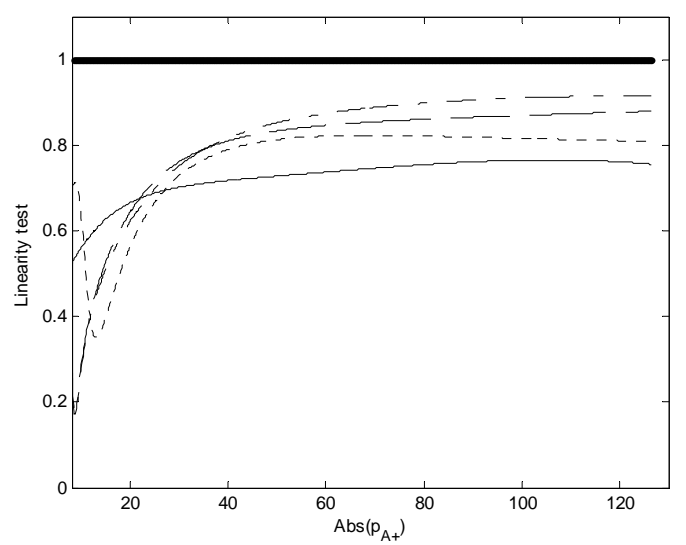

(e)

Figure 8. Linearity tests according to equation (46) for the A-side calculations as function of magnitude of the incident pressure wave: thick solid line $-f$, solid line $-3 f$, dashed line $-5 f$, dashed dotted line $-7 f$, dotted line - $9 f$. (a) No matrix regularization applied, nonlinear scattering matrix model, (b) no matrix regularization applied, PHD model, (c) matrix regularization applied assuming $1 \%$ error in the input data, nonlinear scattering matrix model, (d) matrix regularization applied assuming $1 \%$ error in the input data, PHD model, (e) matrix regularization applied assuming $10 \%$ error in the input data, nonlinear scattering matrix model, (f) matrix regularization applied assuming $10 \%$ error in the input data, PHD model. 


\section{Two-sided nonlinear two-port results}

Nonlinear scattering matrix results for the terms describing the non-linear energy transfer from the excitation frequency to the higher harmonics $S_{A n A l}, S_{A n B 1}, S_{B n A l}$ and $S_{B n B I}$ are presented in Figure 9. These results give information about the levels of the sound transferred to higher harmonics for the tested sample. It can be seen that the level at three times the excitation frequency will be a couple of percent of the excitation level.

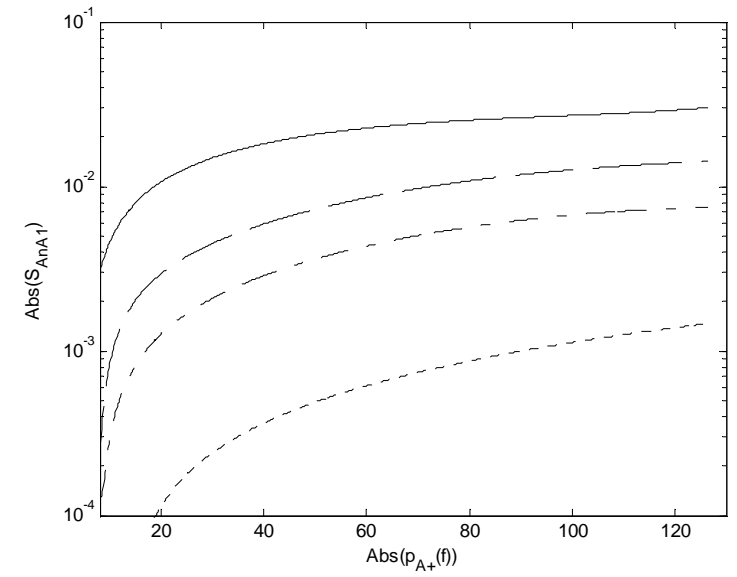

(a)

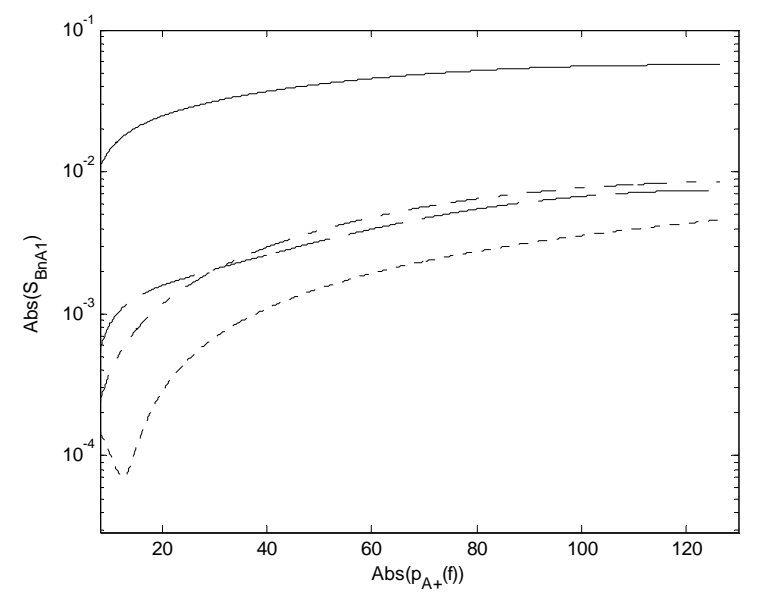

(c)

Figure 9. Absolute value of scattering matrix elements plotted against the magnitude of the incident pressure wave: solid line - $3 f$, dashed line - $5 f$, dashed dotted line - $7 f$, dotted line - $9 f$. (a) $S_{A n A 1}$, (b) $S_{A n B 1}$, (c) $S_{B n A 1}$, (d) $S_{B n B 1}$.

The corresponding results for the PHD model are shown in Figure 10. Here we are only presenting the terms representing energy transfer from the excitation side A since the components representing energy transfer from the B-side are split in two parts in the PHD model. It can be seen that there is a good agreement when comparing $S_{A n A l}$, to $X_{A 1 A 1}$ and $S_{B n A 1}$ to $X_{B n A 1}$.

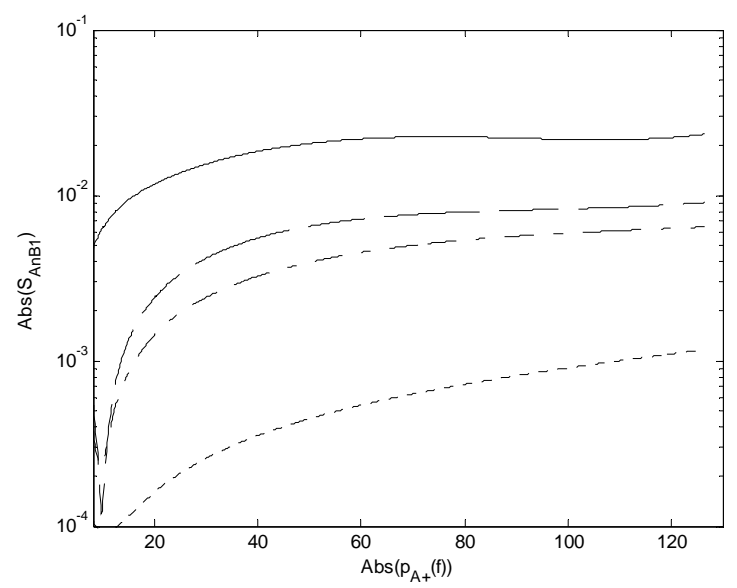

(b)

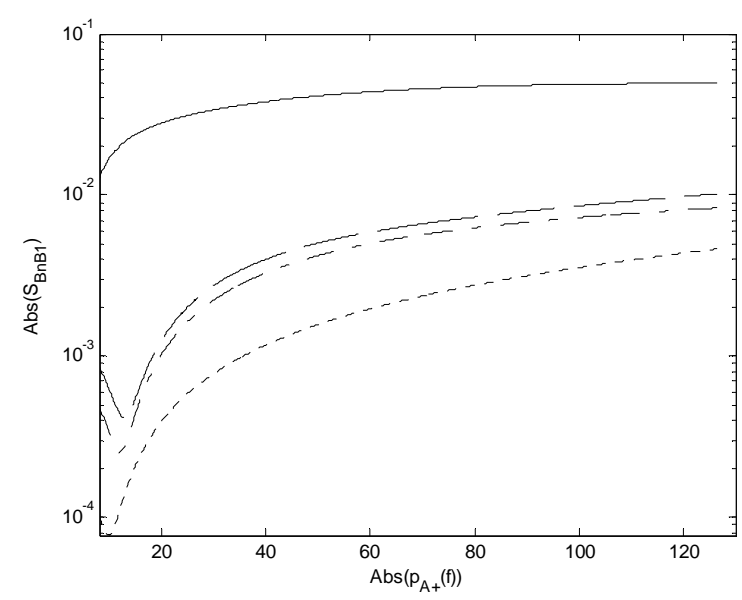

(d) 


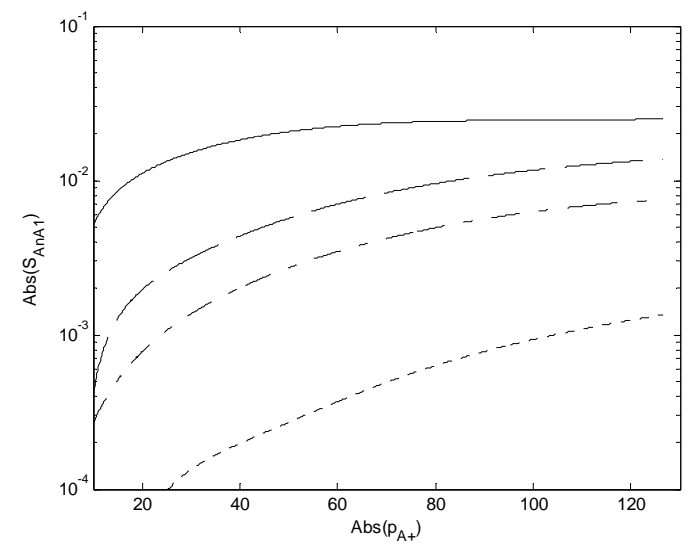

(a)

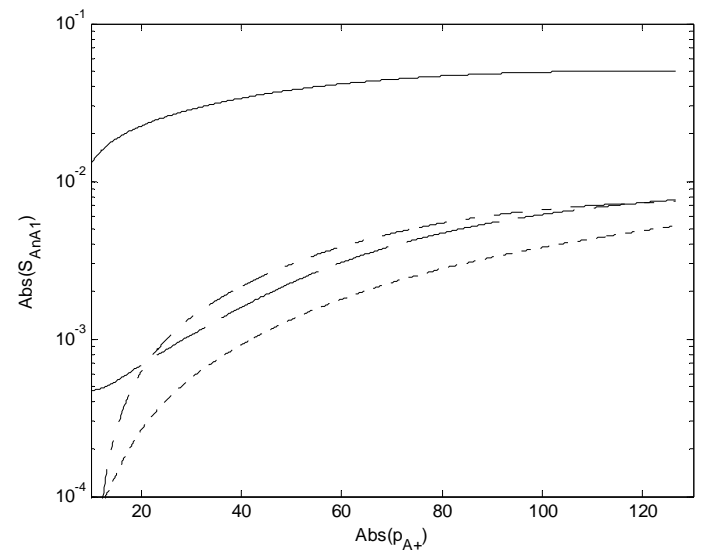

(b)

Figure 10. Absolute value of PHD matrix elements plotted against the magnitude of the incident pressure wave: solid line $-3 f$, dashed line $-5 f$, dashed dotted line $-7 f$, dotted line $-9 f$. (a) $X_{A n A 1}$, (b) $X_{B n A 1}$.

In order to make a further comparison of the outcome of using the PHD model with the nonlinear scattering matrix model normalised residuals according to equation (48) have been calculated. Examples of the results are presented in Figures 11 to 13. It can be seen that the result for the excitation frequency $(f)$ is very similar for the nonlinear scattering matrix and PHD model indicating a residual corresponding to a couple of percent. For the $3 \mathrm{f}$ component we get lower values with the PHD model with $10 \%$ at the highest excitation levels while the nonlinear scattering matrix model gives 20-30\%. The $5 f$ component shows a big difference between the results at the $\mathrm{A}$ and $\mathrm{B}$ sides with a $15 \%$ residual for the PHD model on the A side at the highest excitation levels, while the B side has residual of $100 \%$. The $7 \mathrm{f}$ gave a similar result as the $5 \mathrm{f}$ component for the PHD model while the $9 \mathrm{f}$ component gave a 50-70 \% residual. I all cases except for the excitation frequency the nonlinear scattering matrix model gives higher residuals. It is however to be remembered that there is a higher degree of over-determination for the nonlinear scattering matrix model where we, for components 3f, $5 \mathrm{f}, 7 \mathrm{f}$ and $9 \mathrm{f}$, need 4 loads while we for the PHD model need 7 loads, out of the available 9.

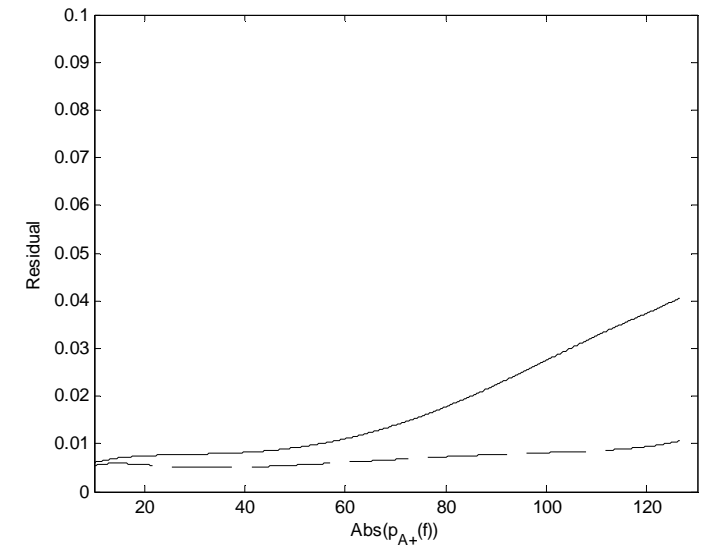

(a)

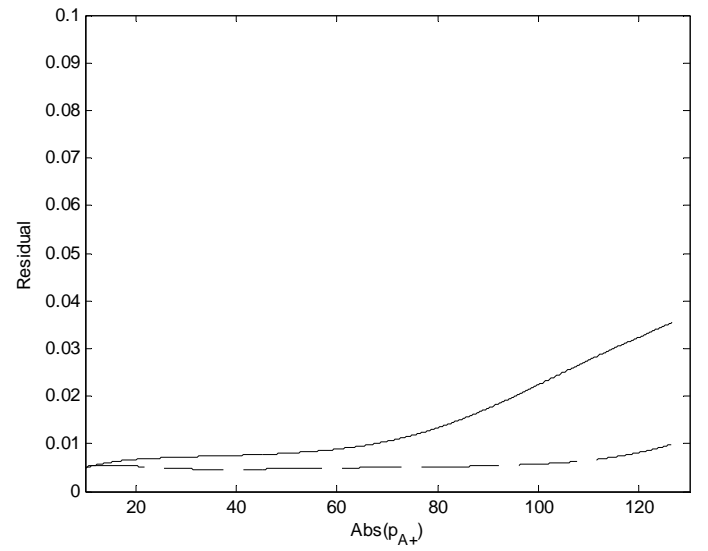

(b)

Figure 11. Residuals for the excitation frequency calculations plotted against the magnitude of the incident pressure wave: solid line - A side, dashed line - B side. (a) Nonlinear scattering matrix model, (b) PHD model. 


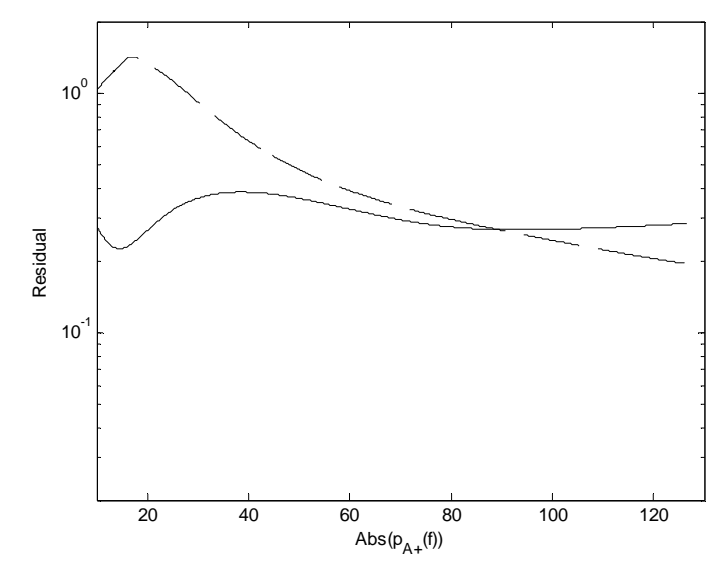

(a)

Figure 12. Residuals for the $3 f$ component calculations plotted against the magnitude of the incident pressure wave: solid line - A side, dashed line - B side. (a) Nonlinear scattering matrix model, (b) PHD model.

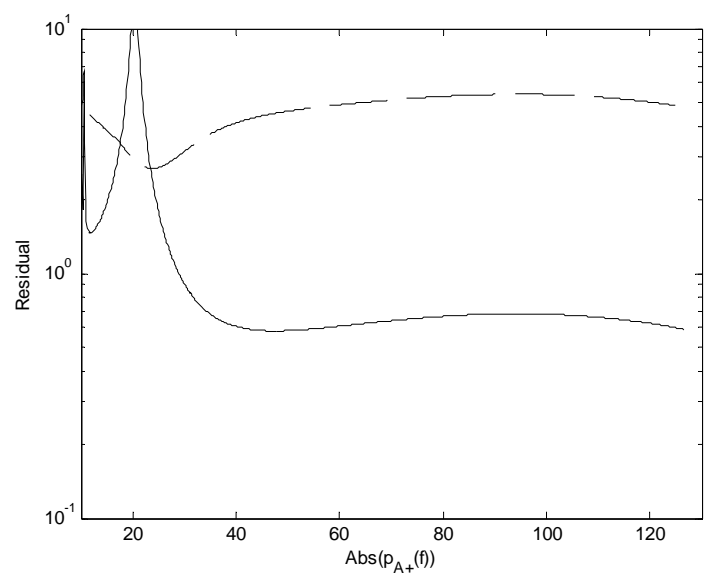

(a)

Figure 13. Residuals for the $5 f$ component calculations plotted against the magnitude of the incident pressure wave: solid line - A side, dashed line - B side. (a) Nonlinear scattering matrix model, (b) PHD model.

Below the experimental results are used to demonstrate how the power relations discussed in section III C can be used. Figure 14 shows reflection factor, transmission factor and absorption factor results for the excitation frequency for three different acoustic load cases compared to the results obtained when calculating the non-reflecting load results using equation (36) to (38). It can be seen that the result as expected is different for the different acoustic loads. It is to be noted that the results for the non-reflecting terminations presented here are based on the nonlinear scattering matrix. Since the difference using the PHD model was small for the $S_{A n A l}, S_{B n A l}$ components which according to equation (36) to (38) are the only components contributing to the result for the non-reflecting termination case the difference in results would be small if the PHD model results were used instead. 


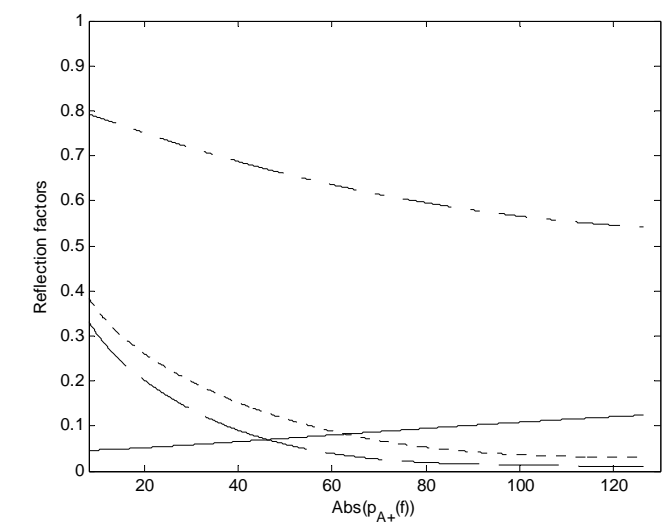

(a)

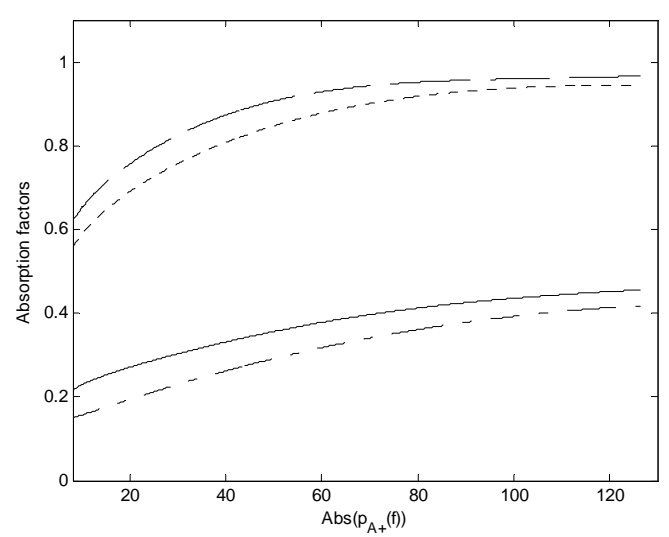

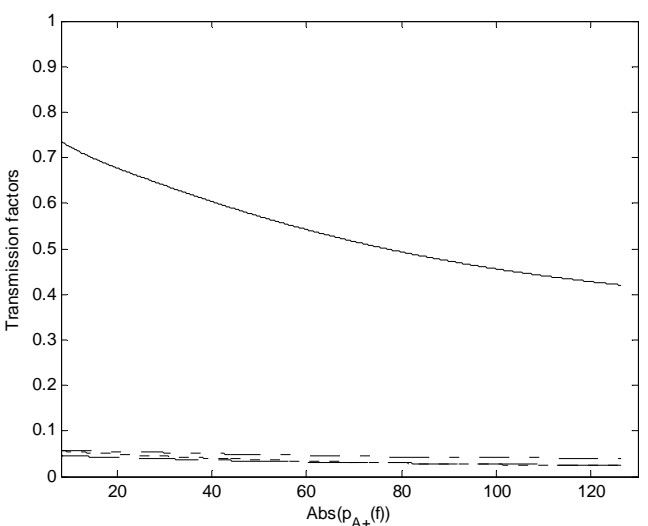

(b)

(c)

Figure 14. Reflection, transmission and absorption factors at the excitation frequency: solid line - reflection free termination, dashed line - termination 1 , dashed-dotted line - termination 4 , dotted line - termination 8. (a) Reflection factor, (b) transmission factor, (c) absorption factor.

Figure 15 shows the results for the frequency component $3 f$. The results are also here different depending on the acoustic loading. It can also be seen that a very small part of the power is transmitted to the $3 f$ harmonic, the order of magnitude is $10^{-3}$ for the non-reflecting termination case, so most of the power not reflected or transmitted at the excitation frequency is lost as heat or vorticity.

The results presented in Figure 14 and 15 illustrates that when a nonlinear scattering matrix model has been obtained through the techniques described here it is possible to predict the sound power generated at the higher harmonics for any combination of terminations. 


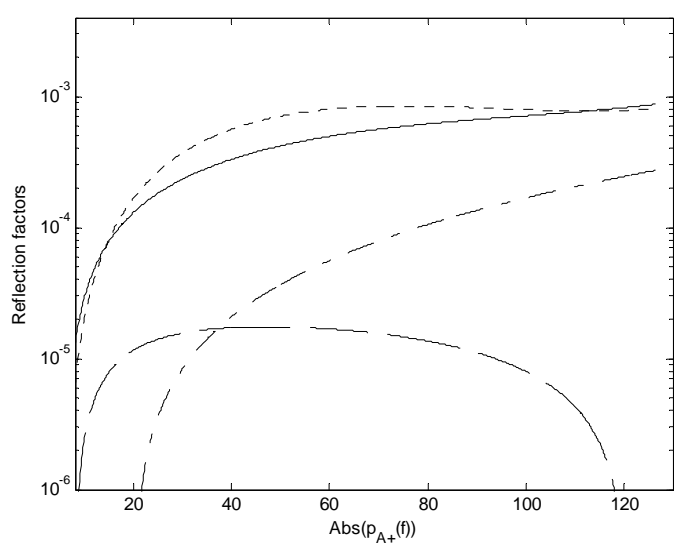

(a) termination, dashed line - termination 1 , dashed-dotted line - termination 4 , dotted line - termination 8 . (a) Reflection factor, (b) transmission factor.

\section{E. One-sided nonlinear two-port results}

Figure 16 shows the absolute value and phase of nonlinear scattering matrix elements $S_{n 1}$ as function of the magnitude of the incident wave. The magnitude of $S_{n 1}$ gives information about the non-linear energy transfer from a pressure wave incident with frequency $f$ to the "transmitted" pressure waves at frequency $3 f, 5 f, 7 f$ and $9 f$.
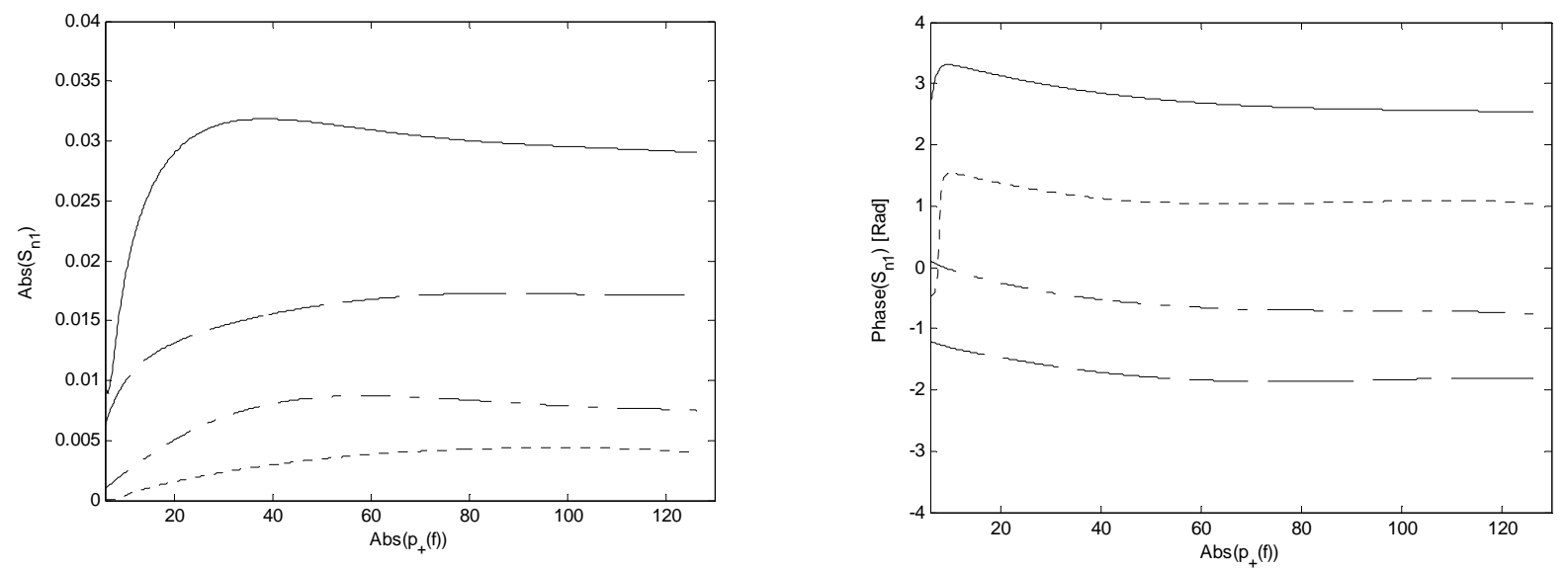

Figure 16. Absolute value and phase of scattering matrix element $S_{n 1}$ plotted against the magnitude of the incident wave: full line - results at $3 f$, dashed line - results at $5 f$, dashed-dotted line - results at $7 f$, dotted line - results at $9 f$.

It can be seen that the magnitude of element $S_{n 1}$ approaches a constant level at high levels of excitation and decreases by approximately a factor 2 for each harmonic, going from 0.03 at $3 f$ to 0.015 at $5 f, 0.0075$ at $7 f$ and 0.0038 at $9 f$. Also the phase seems to exhibit a certain pattern increasing by a factor close to $\pi / 2$ for each harmonic, going from $0.8 \pi$ for $3 f$ to around $-0.6 \pi$ at $5 f,-0.2 \pi$ at $7 f$ and $0.3 \pi$ at $9 f$. Figure 17 shows the data for harmonics $5 f, 7 f$ and $9 f$ shifted by these factors compared to the result at $3 f$. Considering the fact that the experimental error could be $10 \%$ there is a reasonable fit at high levels of excitation. Figure 17 (c) shows the result when the absolute value is instead plotted against the inverse Strouhal number $\left(1 / S t=\left|u_{+}(f)\right| / \omega t\right)$ based on the particle velocity of the incident wave in the holes $\left(\left|u_{+}(f)\right|=\left|p_{+}(f)\right| / \rho_{0} c \sigma C_{D}\right)$ and the plate thickness $(t)$, where $\sigma$ is the percentage open area and $C_{D}$ a discharge coefficient,. The discharge coefficient was set $0.895^{10}$. The inverse Strouhal number formulated in this way relates the particle displacement in the holes to the perforate plate thickness which can be considered as a parameter of relevance for the onset of non-linearity. Plotting against this parameter seems to give a reasonable fit of 
the data obtained for different harmonics. The results in Figure 17 illustrates how the methods developed can be used to increase the understanding of the nonlinear energy transfer to higher harmonics.

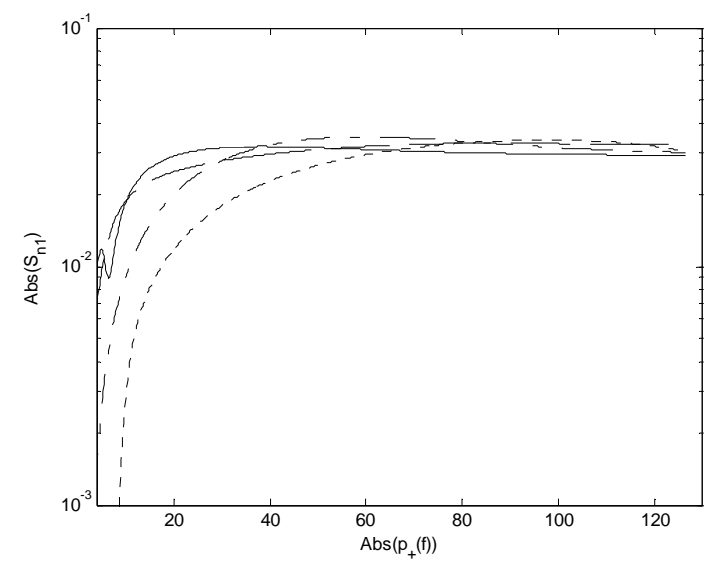

(a)

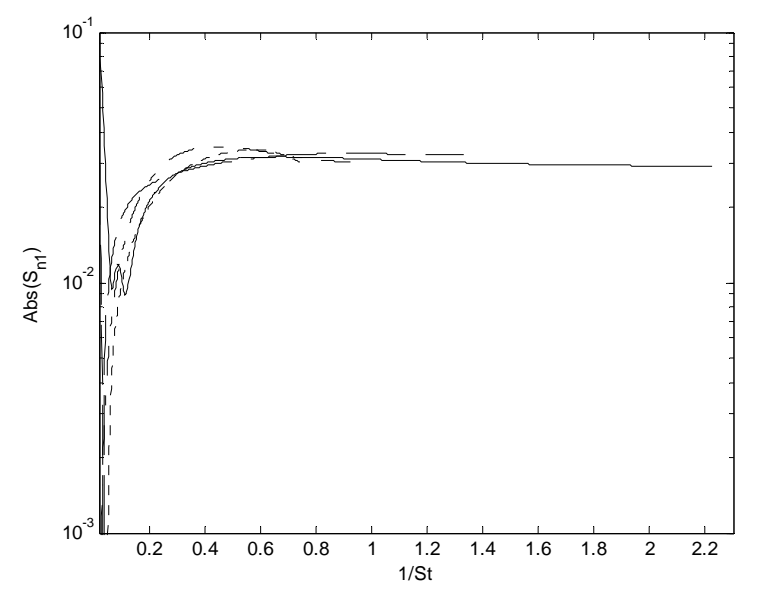

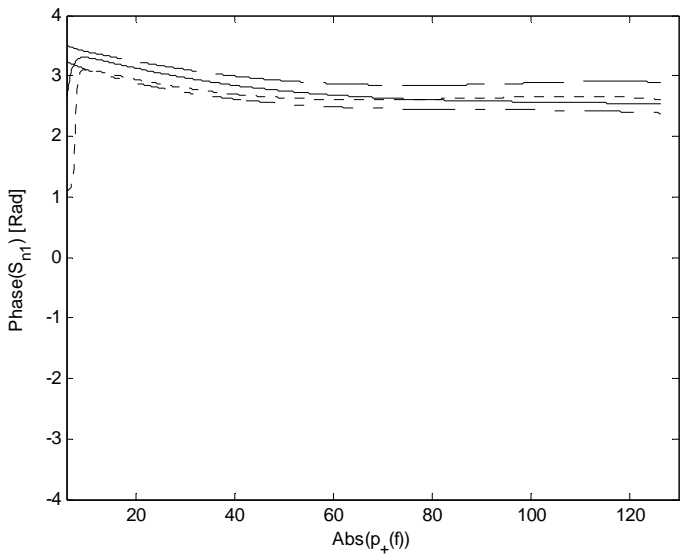

(b)

(c)

Figure 17. Absolute value and phase of scattering matrix element $S_{n 1}$, with the results at $5 f, 7 f$ and $9 f$ shifted: full line - results at $3 f$, dashed line - results at $5 f$, dashed-dotted line - results at $7 f$, dotted line - results at $9 f$. (a) Absolute value plotted against the magnitude of the incident pressure wave, (b) phase plotted against the magnitude of the incident pressure wave, (c) Absolute value plotted against inverse Strouhal number.

Figure 18 shows the absolute value and phase of $S_{n n}$ estimated using equation (40) as a function of the magnitude of the incident wave. $S_{n n}$ gives information about the reflection of acoustic waves at the higher harmonics. The magnitude should therefore be smaller than one. If the assumption that the level of excitation at the higher harmonics is small so that we are in the linear regime the $S_{n n}$ data should also be independent of the level of excitation at the fundamental frequency. This is reasonably true for the data presented in Figure 18. This is further confirmed by the comparison, in Figure 19, for the $3 f$ component with the directly measured reflection coefficient at $3 f$ using low level excitation. 

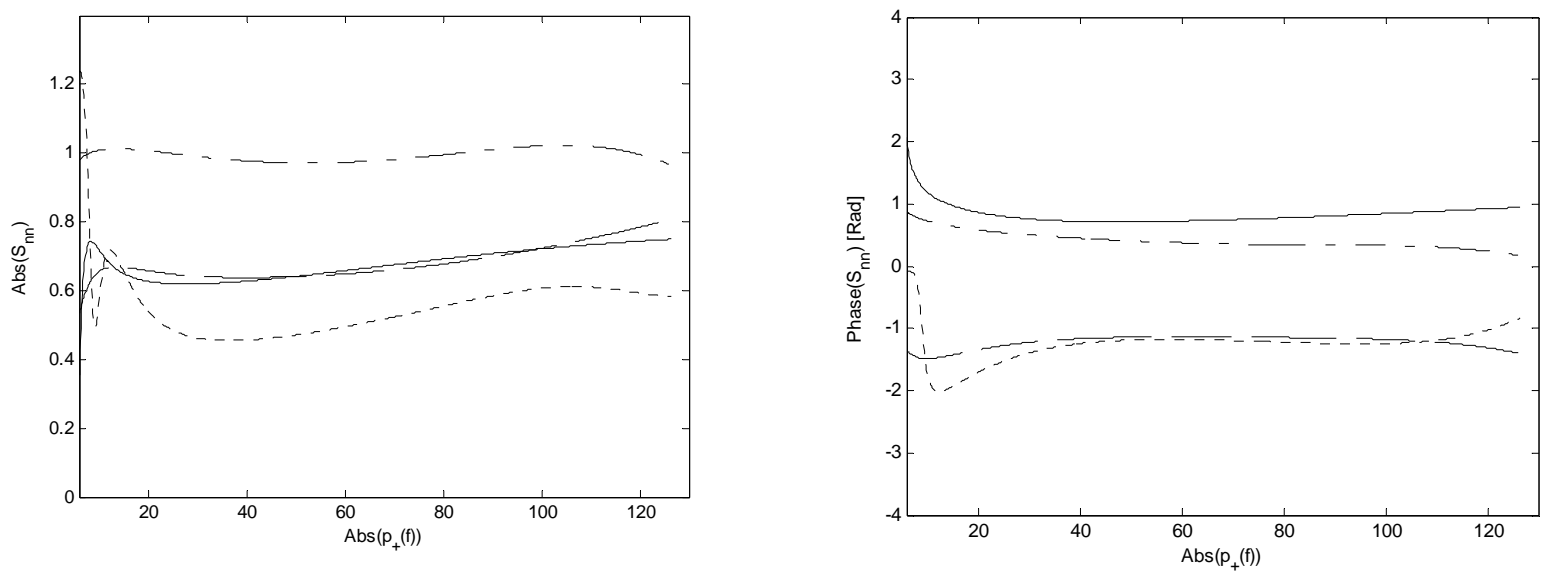

Figure 18. Absolute value and phase of scattering matrix element $S_{n n}$ plotted against the magnitude of the incident pressure wave: full line - results at $3 f$, dashed line - results at $5 f$, dashed-dotted line - results at $7 f$, dotted line - results at $9 f$.
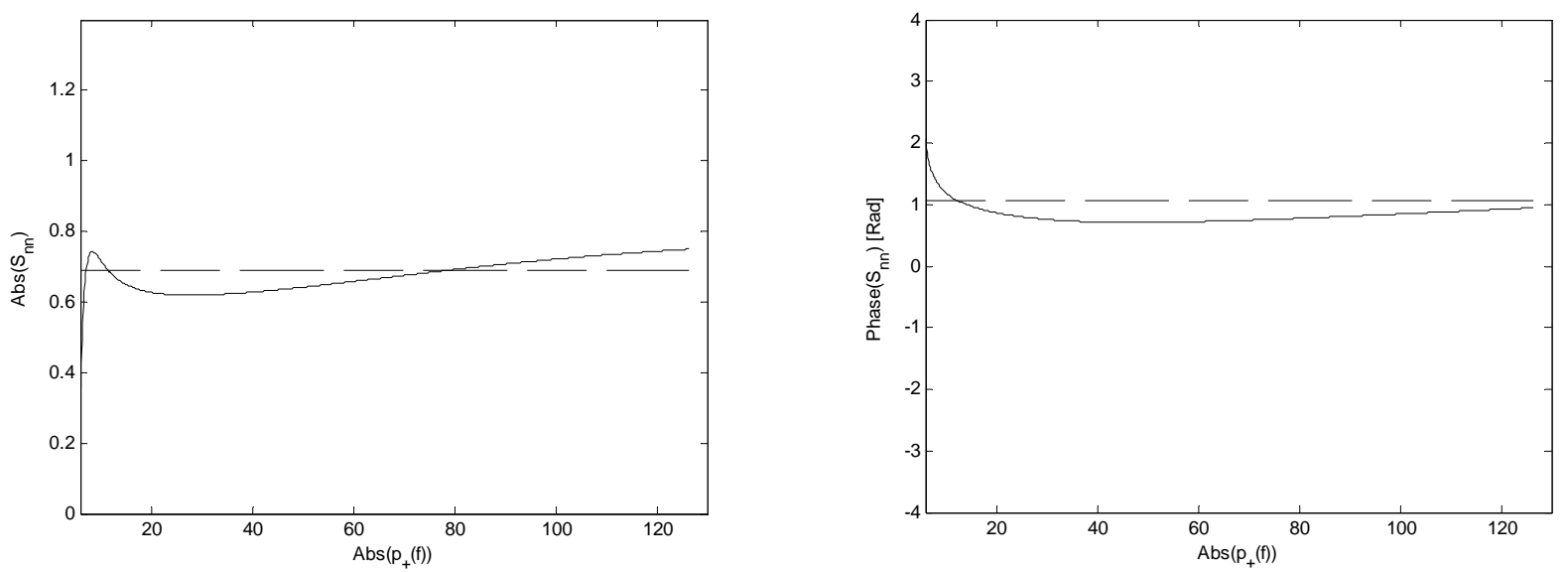

Figure 19. Absolute value and phase of scattering matrix element $S_{33}$ plotted against the magnitude of the incident pressure wave: full line $-S_{33}$ obtained using nonlinear scattering matrix, dashed line - results obtained using direct reflection coefficient measurement, according to.

Having established that $S_{n n}$ can be replaced by the reflection coefficient measured separately with low level excitation it is finally possible to estimate $S_{n 1}$ using this information. The result for the $3 f$ component is presented in Figure 20 compared to result obtained using the full nonlinear scattering matrix, showing a good agreement. 

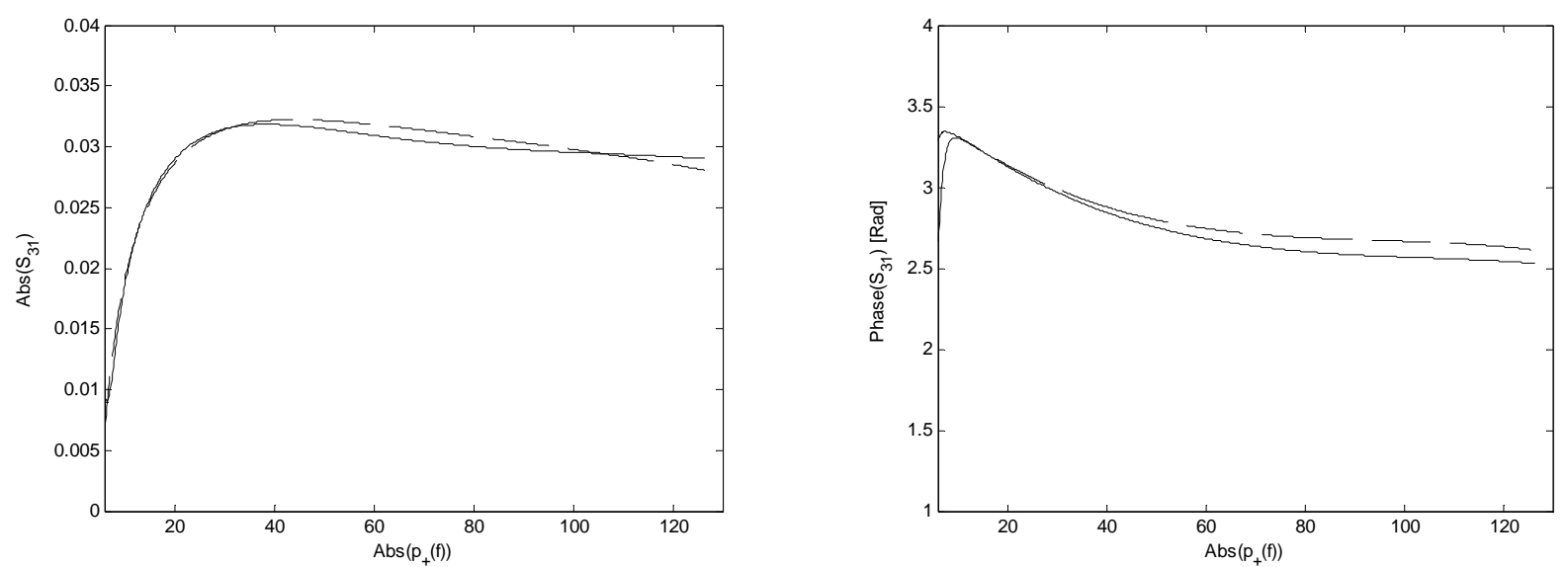

Figure 20. Absolute value and phase of scattering matrix element $S_{31}$ plotted against the magnitude of the incident pressure wave: full line $-S_{31}$ obtained using full nonlinear scattering matrix, dashed line $-S_{31}$ obtained using using $S_{33}$ estimated from separate low level measurement.

The conclusions which can be drawn from the results presented in this section are: that the nonlinear scattering matrix does give reasonable results, that the assumption about replacing the $S_{n n}$ component with the measured low level reflection coefficient is valid and that the results can be used to make interesting observations regarding the nonlinear energy transfer from the excitation frequency to the higher order odd harmonics.

Figure 21 shows the absolute value an phase of elements $X_{n 1}$ as an example of the results of applying the polyharmonic distortion (PHD) model. The amplitude should be compared to the amplitude of element $S_{n 1}$ from the nonlinear scattering matrix results presented in Figure 16. The agreement is fairly good even though there are some more oscillations in the $X_{n 1}$ results.
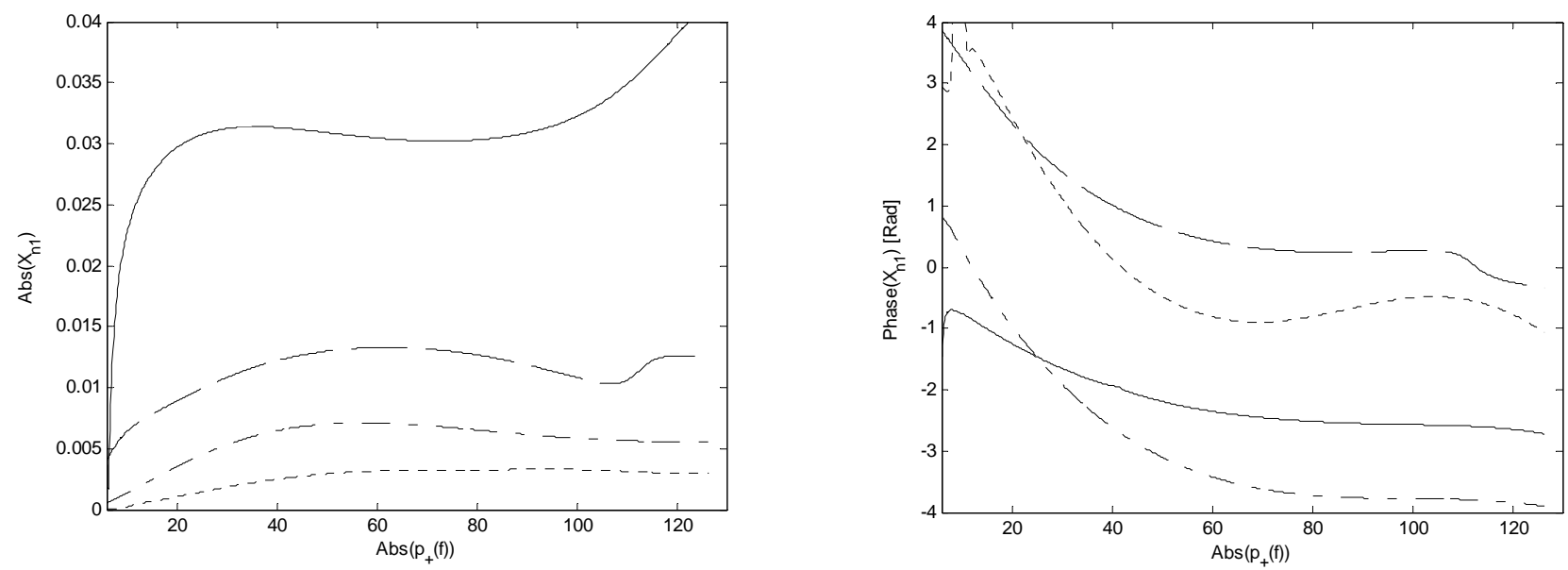

Figure 21. Amplitude and phase of PHD model elements $X_{n 1}$ as function of the magnitude of the incident pressure wave: solid line - results at $3 f$, dashed line - results at $5 f$, dashed-dotted line - results at $7 f$, dotted line - results at $9 f$.

The effect of errors in the input data on the calculated results has also been estimated through numerical simulation. An error in the measured travelling wave pressure amplitudes of $1 \%$ has been used and the results of 50 different combinations of phase angles for these errors has been calculated. Figures 22 and 23 show the result for the $S_{n 1}$ and $X_{n 1}$ elements in the nonlinear scattering matrix and the PHD model. Figures 24 and 25 show the corresponding results for the $S_{n n}$ an $X_{n n}$ elements. The curve for each scattering matrix element has been plotted surrounded by curves representing plus and minus one standard deviation. It can be seen that the error is larger for the $S_{n n}$ elements compared to the $S_{n 1}$ elements. The relative errors are also smallest for the $3 f$ and $5 f$ components as expected with around 1-4 \% error in $S_{n 1}$ for 1\% error in the input data. For the PHD model it can be seen that the 
errors in the results are generally higher compared to the results for the nonlinear scattering matrix. The errors for $X_{n 1}$ is, for instance, $8-10 \%$ at $3 f$, growing to $15-20 \%$ at $5 f$ and $7 f$ and around $40 \%$ at $9 f$.

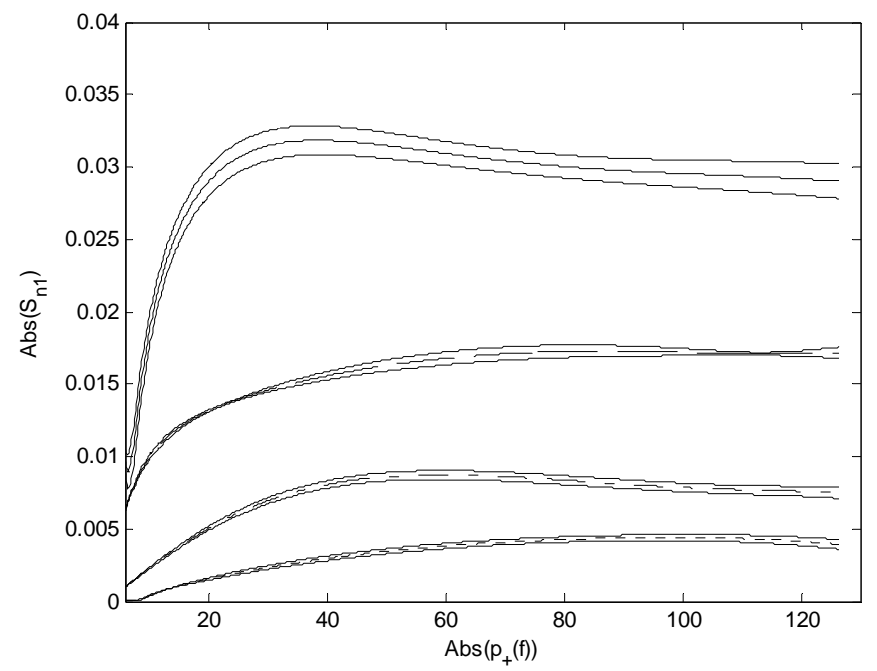

Figure 22. Scattering matrix elements $S_{n 1}$ with error estimates in the form of plus or minus one standard deviation for $1 \%$ error in the input data as function of the magnitude of the incident pressure wave: solid line - results at $3 f$, dashed line - results at $5 f$, dashed-dotted line - results at $7 f$, dotted line - results at $9 f$.

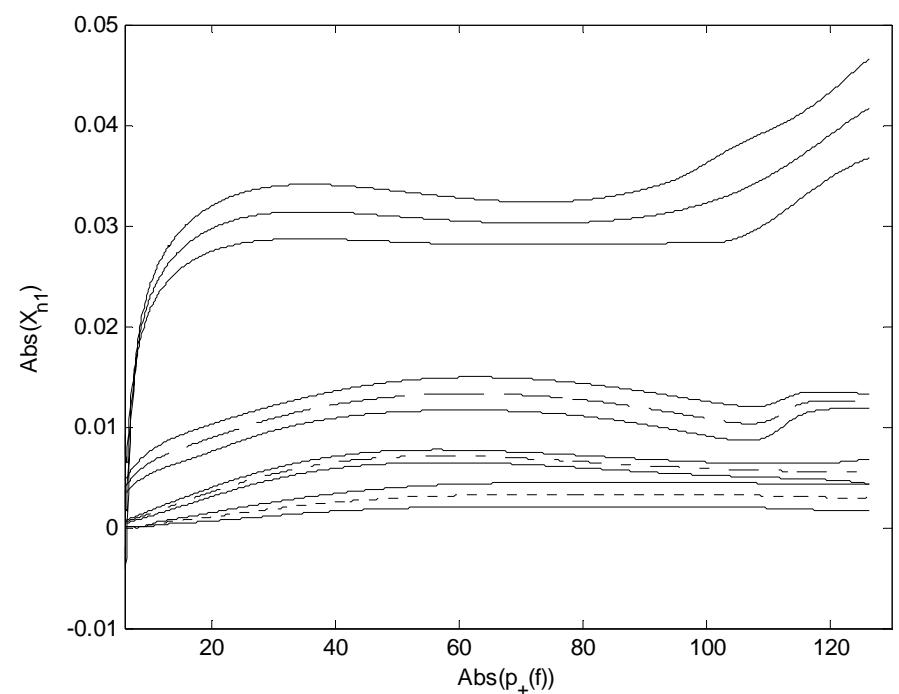

Figure 23. PHD model elements $X_{n 1}$ with error estimates in the form of plus or minus one standard deviation for $1 \%$ error in the input data as function of the magnitude of the incident pressure wave: solid line - results at $3 f$, dashed line - results at $5 f$, dashed-dotted line - results at $7 f$, dotted line - results at $9 f$. 

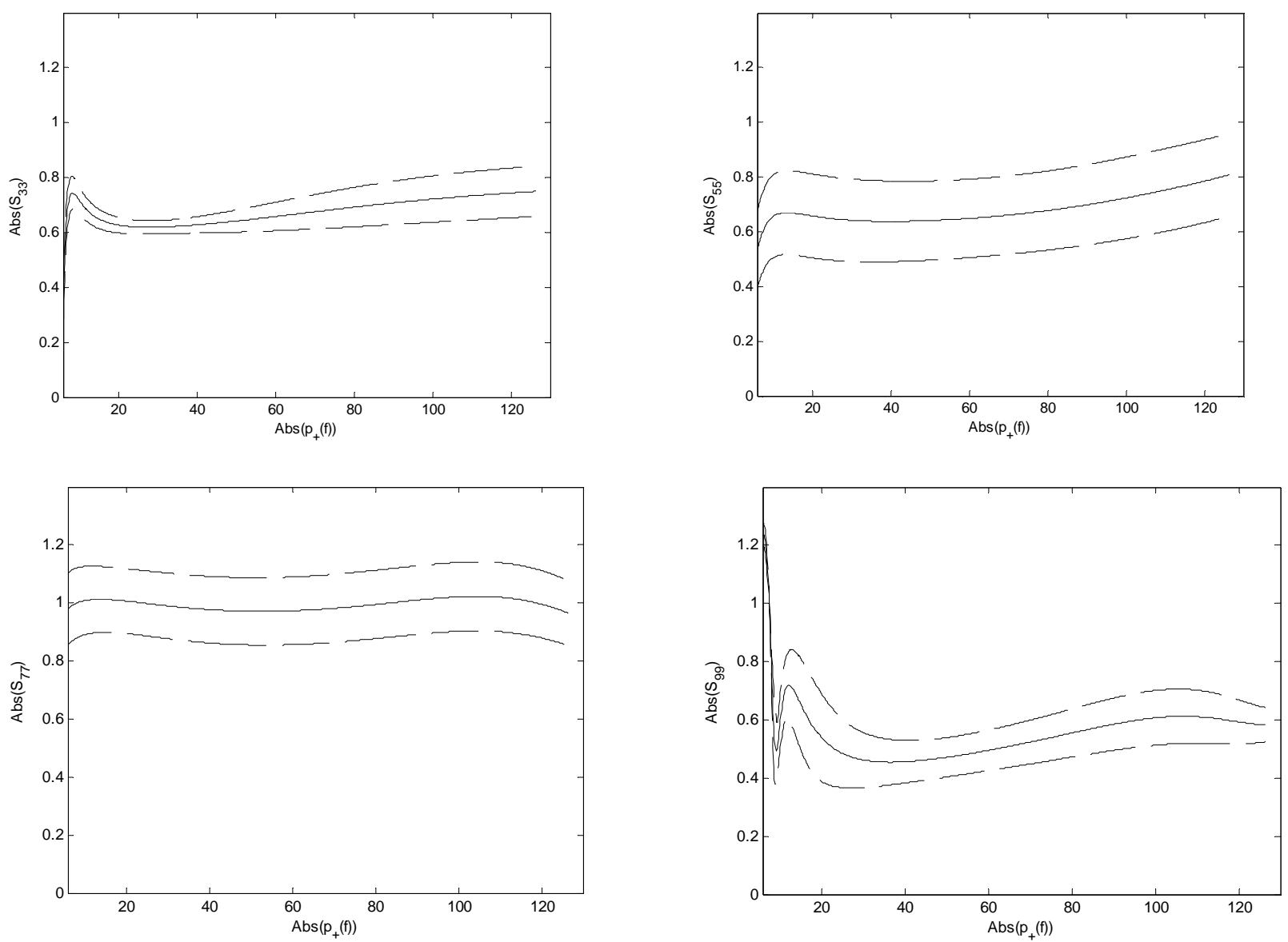

Figure 24. Scattering matrix elements $S_{n n}$ with error estimates in the form of plus or minus one standard deviation for $1 \%$ error in the input data as function of the magnitude of the incident pressure wave: solid line - results at $3 f$, dashed line - results at $5 f$, dashed-dotted line - results at $7 f$, dotted line - results at $9 f$. 


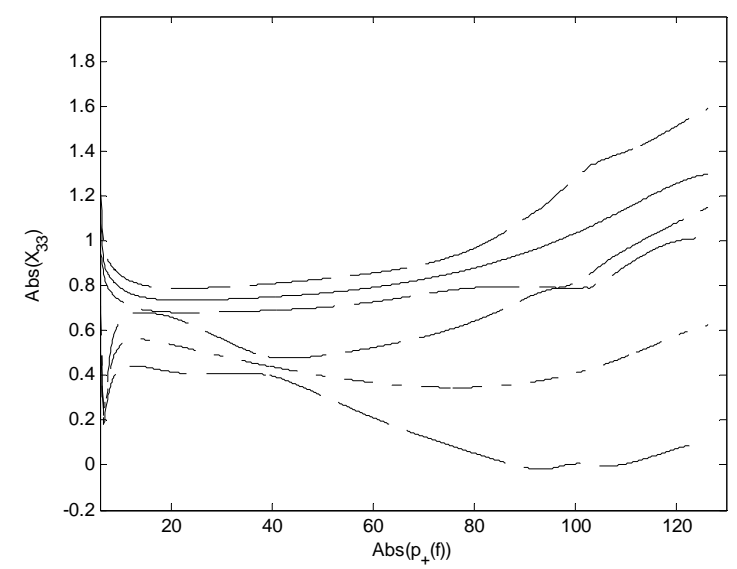

(a)

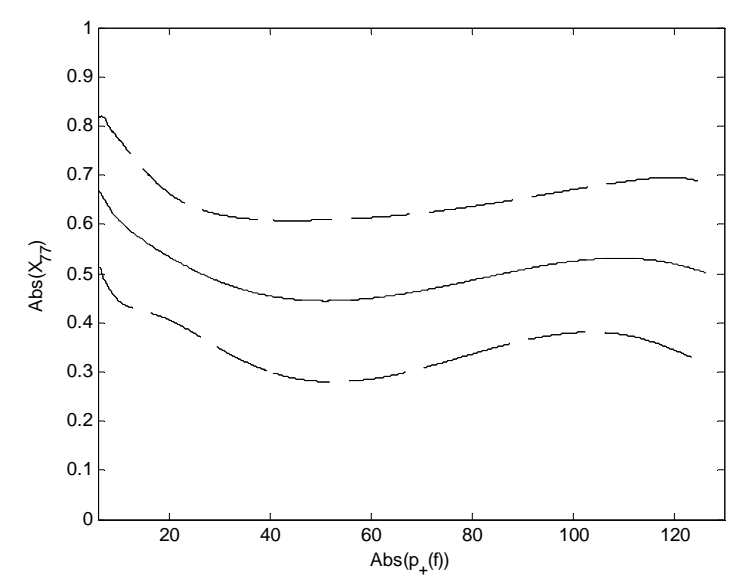

(c)

Figure 25. PHD distortion model elements $X_{n n}$ and $\Xi_{n n}$ with error estimates in the form of plus or minus one standard deviation for $1 \%$ error in the input data as function of the magnitude of the incident pressure wave: solid line $-X_{n n}$, dashed line $-\Xi_{n n}$. (a) Results for $3 f$, (b) results for $5 f$, (c) results for $7 f$, (d) results for $9 f$.

\section{Conclusions}

In the present paper measurements of non-linear acoustic properties of in-duct samples, such as perforates occurring in aircraft engine liners and automotive mufflers, have been discussed. A number of novel two-port models applicable for single frequency excitation have been suggested and tests were made for a perforate sample. In order to solve for the parameters of the models tested it was necessary to do measurements for a number of independent acoustic test cases. This was achieved by changing the terminations in the impedance tube. The level of excitation was varied to study the nonlinear effects and, to get valid experimental data, it is important that he level of excitation is the same for each acoustic load used in the test. A method to align the measured data for the different loads using a polynomial fit was therefore developed. The theory of polyharmonic distortion modelling used in commercial analysers for nonlinear characterisation of microwave systems has been introduced in acoustics. It was found that both the nonlinear scattering matrix formulation and the polyharmonic distortion model when applied to the sample tested gave acceptable results for describing the nonlinear energy transfer between a fundamental frequency and the first odd harmonics. The assumption that energy transfer only takes place from the excitation frequency to the odd harmonics and not from higher harmonics back to lower frequencies was shown to be valid. This leads to a formulation of the nonlinear two-port models where coupling between the fundamental excitation frequency and each odd higher harmonic could be treated separately, reducing the size of the system to be identified.

American Institute of Aeronautics and Astronautics 
The nonlinear high level forcing from one side of the sample introduces a system un-symmetry. It was shown how it is possible to use the two-sided nonlinear two-port model to predict the energy transfer to higher harmonics for any acoustic termination applied to either side of the sample. As an example the case of perfectly non-reflecting terminations was used. For the one-sided nonlinear scattering matrix, plotting elements $S_{n l}$, describing energy transfer from the fundamental frequency $(f)$ to the higher harmonics (nf), against an inverse Strouhal number based on the particle velocity in the incident wave and the perforate plate thickness gave a collapse of the data. This illustrates that the inverse Strouhal number is one parameter controlling the non-linearity. It was also shown that the nonlinear scattering matrix gives reasonable results and that the simplifying assumption about replacing the $S_{n n}$ components with directly measured low level reflection coefficients at the frequencies of the higher harmonics is valid. The error analysis showed that the PHD model results had larger errors. No clear advantage of using the PHD model over the nonlinear scattering matrix model could therefore be seen even though it rests on a more solid theoretical foundation. It is the hope that these technique in the future can be used to analyse nonlinear interaction for multi-tone excitation, which is what we often have in practical applications. In these practical applications there would also usually be mean flow which will change the situation. It is however hoped that this study could be a first step to also understand the combination of mean flow and high level acoustic excitation. In the practical applications mentioned above it is not uncommon that the mean flow level and the local particle velocities in the perforate holes are of the same order of magnitude.

\section{References}

${ }^{1}$ Bodén, H., "Experimental investigation of harmonic interaction effects for perforates”,

AIAA Paper 2005- 2896.

${ }^{2}$ Bodén, H., Ying, G. and Tözün, H.B., "Experimental investigation of nonlinear acoustic properties for perforates”, AIAA Paper 2006- 2404.

${ }^{3}$ Bodén, H., “Acoustic characterization of perforates using on-linear system identification techniques”, AIAA Paper 2007- 3530.

${ }^{4}$ Bodén, H., “On impedance measurements for samples with non-linear acoustic properties”, AIAA Paper 2008- 3016.

${ }^{5}$ Bodén, H., "Multi-port techniques for acoustic characterisation of non-linear in-duct discontinuities."

AIAA Paper 2009- 3106.

${ }^{6}$ Ingård, U. and Labate, S., "Acoustic circulation effects and the nonlinear impedance of orifices", Journal of the Acoustical Society of America, Vol. 22, 1950, pp. 211-219.

7Ingård, U. and Ising, H., "Acoustic nonlinearity of an orifice", Journal of the Acoustical Society of America,

Vol. 42, 1967, pp. 6-17.

${ }^{8}$ Melling, T.H., "The acoustic impedance of perforates at medium and high sound pressure levels", Journal of Sound and Vibration, Vol. 29, No. 1, 1973, pp. 1-65.

${ }^{9}$ Elnady, T. and Bodén, H., "On semi-empirical liner impedance modeling with grazing flow", AIAA Paper, AIAA 2003-3304, May 2003.

${ }^{10}$ Elnady, T., "Modelling and characterization of perforates in lined ducts and mufflers (Paper III)", PhD Thesis, Department of Aeronautical and Vehicle Engineering, KTH, Stockholm, Sweden, 2004.

${ }^{11}$ Ingård, U., "Nonlinear distortion of sound transmitted through an orifice", Journal of the Acoustical Society of America, Vol. 48, 1970, pp. 32-33.

${ }^{12}$ Tam, C.K.W. and Kurbatski, K.A., "Micro-fluid dynamics and acoustics of resonant liners”, AIAA Paper, AIAA 99-1850, 1999.

${ }^{13}$ Tam, C.K.W., Kurbatski, K.A., Ahuja K.K.and Gaeta Jr., R.J. “A Numerical and Experimental Investigation of the Dissipation Mechanisms of Resonant Acoustic Liners”, Journal of Sound and Vibration, Vol. 245, No. 3, 2001, pp. 545-557.

${ }^{14}$ Tam, C.K.W., Ju, H., Jones, M.G, Watson, W.R. and Parrott, T.L. “A Computational and Experimental Study of Slit Resonators”, Journal of Sound and Vibration, Vol. 284, No. 3-5, 2005, pp. 947-984.

${ }^{15}$ Sippilä, M., Lehtinen, K. and Porra, V. "High-frequency periodic time domain waveform measurement system," IEEE Trans.Microwave Theory Tech., vol. 36, pp. 1397-1405, Oct. 1988.

${ }^{16}$ Lott, U. "Measurement of magnitude and phase of harmonics generated in nonlinear microwave two-ports," IEEE

Trans.Microwave Theory Tech., vol. 37, pp. 1506-1511, Oct. 1989.

${ }^{17}$ Verspecht, J., Debie, P. Barel, A. and Martens, L. "Accurate on wafer measurements of phase and amplitude of the spectral components of incident and scattered voltage waves at the signal ports of a nonlinear microwave device," in 1995 IEEE MTT-S Int.Microwave Symp. Dig., May 1995, vol. 3, pp. 1029-1032.

${ }^{18}$ Verspecht, J., “Large-Signal Network Analysis”, IEEE Microwave Magazine, Vol. 6, Issue 4, December 2005, pp. 82-92.

${ }^{19}$ Verspecht, J. and Root D.E., "Polyharmonic Distortion Modeling”, IEEE Microwave Magazine, June 2006, pp. 44-57.

${ }^{20}$ Vye, D., "Fundamentally Changing Nonlinear Microwave Design", Microwave Journal, March 2010, Cover Feature, pp. 22-39.

${ }^{21}$ Åbom, M., "Measurement of the scattering matrix of acoustical two-ports", Mechanical Systems and Signal Processing, Vol. 5, 1991, pp. 89-104. 
${ }^{22}$ Chung, J.Y. and Blaser, D.A., "Transfer function method of measuring in-duct acoustic properties", Journal of the Acoustical Society of America, Vol. 68, 1980, pp. 907-921.

${ }^{23}$ Seybert, A.S. and Ross, "Experimental determination of acoustic properties using a two-microphone random-excitation technique", Journal of the Acoustical Society of America, Vol. 61, 1977, pp. 1362-1370.

${ }^{24}$ Albertsson, F. and Bodén H., "Linearity tests for in-duct acoustic one-port sources," Journal of Sound and Vibration, Vol. 237, No. 1, 2000, pp. 45-65.

${ }^{25}$ Bodén H.and Åbom, M., "Influence of errors on the two microphone method for measuring acoustic properties in ducts," Journal of Acoustical Society of America, Vol. 79, 541-549 (1986)

${ }^{26}$ Åbom M. and Bodén H., "Error analysis of two-microphone measurements in ducts with flow," Journal of Acoustical Society of America, Vol. 83, pp. 2429-2438 (1988) 